\title{
BIREGULAR MODELS OF LOG DEL PEZZO SURFACES WITH RIGID SINGULARITIES
}

\author{
MUHAMMAD IMRAN QURESHI
}

\begin{abstract}
We construct biregular models of families of log Del Pezzo surfaces with rigid cyclic quotient singularities such that a general member in each family is wellformed and quasismooth. Each biregular model consists of infinite series of such families of surfaces; parameterized by the natural numbers $\mathbb{N}$. Each family in these biregular models is represented by either a codimension 3 Pfaffian format modelled on the Plücker embedding of $\operatorname{Gr}(2,5)$ or a codimension 4 format modelled on the Segre embedding of $\mathbb{P}^{2} \times \mathbb{P}^{2}$. In particular, we show the existence of two biregular models in codimension 4 which are bi parameterized, giving rise to an infinite series of models of families of log Del Pezzo surfaces. We identify those biregular models of surfaces which do not admit a $\mathbb{Q}$-Gorenstein deformation to a toric variety.
\end{abstract}

\section{INTRODUCTION}

A $\log$ Del Pezzo surface $X$ is a projective surface $X$ with $-K_{X}$ ample and having isolated cyclic quotient singularities; such surfaces are also referred to as orbifold Del Pezzo surfaces with isolated orbifold points. The log Del Pezzo surfaces form an interesting class of surfaces which appear naturally in various contexts including the minimal model program [KMM87]. Recently, the construction and classification of orbifold Del Pezzo surfaces have arisen in the mirror symmetry program of Coates-Corti et al $\left[\mathrm{CCG}^{+} 12\right.$ formally conjectured in $\left[\mathrm{ACC}^{+} 16\right]$ for orbifold Del Pezzo surfaces. Such surfaces with $m \times \frac{1}{3}(1,1)$ points have been classified by Corti and Heuberger in [CH17. The classification with a single orbifold point of type $\frac{1}{r}(1,1)$ is provided by Cavey and Prince [CP17]. The log Del Pezzo surfaces have also been studied from the point of view of the existence of orbifold Kahler-Einstein metric on such surfaces in CS13, CPS10, KP15, starting with [JK01]. In [JK01, Johnson and Kollár determined the complete list of del Pezzo hypersurfaces of index 1 in three-dimensional weighted projective spaces, admitting a Kahler-Einstein metric. In CS13, Cheltsov and Shramov classified the Del Pezzo hypersurfaces of index 1 in a weighted projective space satisfying certain condition on their log-canonical threshold. The wellformed and quasismooth weighted complete intersection Del Pezzo surfaces have been classified by Mayanskiy in May16.

We construct biregular models of log Del Pezzo surfaces of Fano index $I=1,2$ in codimension 3 and 4 which are not complete intersections. Each biregular model consists of an infinite series of families of wellformed and quasismooth log Del Pezzo surfaces. Their equations can be described by maximal Pfaffians of a $5 \times 5$ skew symmetric matrix

2010 Mathematics Subject Classification. Primary 14J10, 14M07, 14J45, 14 Q10.

Key words and phrases. log Del Pezzo surface, weighted $\operatorname{Gr}(2,5)$, weighted $\mathbb{P}^{2} \times \mathbb{P}^{2}$, Gorenstein format. 
of forms or $2 \times 2$ minors of the size 3 matrix of forms. We also compute their invariants like plurigenera, degree of the canonical class etc. We identify those families of surfaces which do not admit a $\mathbb{Q}$-Gorenstein deformation to a toric variety. Moreover, we also construct several other families of wellformed and quasismooth log Del Pezzo surfaces in codimension 3 and 4.

Any normal surface $X$ with cyclic quotient singularities admits a $\mathbb{Q}$-Gorenstein partial smoothing to a surface with only rigid singularities by [KSB88]. So we only concentrate on those surfaces having quotient singularities which are rigid under $\mathbb{Q}$-Gorenstein smoothing. We give a complete classification of such log Del Pezzo surfaces up to certain values of a parameter called the adjunction number of the free resolution of its corresponding graded ring, following [BKZ, Qur17a]. This is equivalent to finding all possible families $\mathcal{X} \subset \mathbb{P}\left(a_{i}\right)$ with $\sum a_{i} \leq q+I$, where $q$ is the adjunction number and $I$ is the Fano index of $\mathcal{X}$. It is not a complete list of such varieties which can be constructed using these two Gorenstein formats as the adjunction number $q$ is unbounded. On the other hand the computational evidence suggest that our list of biregular models is complete.

We consider these families of log Del Pezzo surfaces as regular pullbacks (see [BKZ]) from key varieties of weighted Grassmannian w $\mathrm{Gr}(2,5)$ and $\mathrm{w}\left(\mathbb{P}^{2} \times \mathbb{P}^{2}\right)$. They also have a description as complete intersections in some weighted Grassmannian w $\mathrm{Gr}(2,5)$ or in the Segre embedding of weighted $\mathbb{P}^{2} \times \mathbb{P}^{2}$ or in the projective cone over either of those ambient varieties, following Qur17a. We exploit the latter description in the proofs. The most difficult part in our proofs is the singularity analysis of these families. In the case of orbifold Del Pezzo surfaces which are complete intersection in weighted projective space, the quasismoothness can be proved by using criterion given by Fletcher [IF00]. But we do not have a straight forward criteria in higher codimension and we have to prove it case by case. Thus the main challenging part of the computation is to prove the quasismoothness of these models. The invariants like $h^{0}(-K)$ and $-K^{2}$ can be calculated by using the Hilbert series of these biregular models. The Hilbert series also helps us to identify those families which do not admit a $\mathbb{Q}$-Gorenstein deformation to a toric variety. Our motivation is to provide a vast testing ground for the questions like studying the existence of an orbifold Kahler-Einstein metric on a Del Pezzo surface or studying the mirror symmetry conjectures of $\left[\mathrm{ACC}^{+} 16\right]$.

Summary and Results. $\$ 2$ consists of the preliminary and background material for the proofs in the rest of the sections. We recall the definition of a $T$-singularity, a $R$-singularity and the log Del Pezzo surface. We also introduce the notion of model of log Del Pezzo surfaces and give a characterization for a quotient singularity $\frac{1}{r}(a, b)$ to be an $R$-singulary in the context of this article. At the end, we give a general strategy of the proofs coming in the coming sections.

In 93 we construct biregular models of families of orbifold Del Pezzo surfaces in codimension 3 which are the regular pullbacks from the key variety ${ }^{6} \operatorname{Gr}(2,5)$ which we refer to as Pfaffian models. We recall the definition of weighted Grassmannian ${ }^{6} \operatorname{Gr}(2,5)$ and the formula for Hilbert Series from [CR02]. We describe how to compute the degree of the canonical class $-K^{2}$ of a $\log$ Del Pezzo surface appearing in Pfaffian models. The 
main part of this section gives the proof of Theorem 1.1 where we show the existence, wellformedness and quasismoothness of each model. In total we construct eight biregular models, four of them have Fano index 1 and four have index 2. In fact, we get 9 models but one of the index 2 models is not quasismooth which we briefly discuss in 3.2 .

Theorem 1.1. There are 8 biregular models (infinite series) of families of log Del Pezzo surfaces with rigid singularities such that a general member of each family in each model is wellformed and quasismooth with the basket of singularities and invariants given in Table 1. There equations are given by maximal Pfaffians of the $5 \times 5$ skew symmetric matrix of forms giving the embedding of each family $\mathcal{X} \hookrightarrow \mathbb{P}^{5}(a, \ldots, f)$. Moreover, at least 2 of these models do not admit a $\mathbb{Q}$-Gorenstein deformation to a toric variety.

Table 1: Biregular log Del Pezzo Models in Pfaffian format where $q=r-1, s=r+1, t=r+2, u=r+3, v=2 r+1, y=$ $2 r-2, z=2 r-1, m=3 r-2$.

\begin{tabular}{|c|c|c|c|c|c|c|c|c|}
\hline Model & WPS \& Param & Basket $\mathcal{B}$ & $-K^{2}$ & $h^{0}(-K)$ & \multicolumn{4}{|c|}{ Weight Matrix } \\
\hline $\mathrm{Pf}_{11}$ & $\begin{array}{c}\mathbb{P}\left(1^{3}, r^{2}, z\right) \\
r \geq 2 \\
w=\frac{1}{2}(1,1,1, z, z)\end{array}$ & $\frac{1}{z}(1,1)$ & $\frac{2 r+3}{2 r-1}$ & 3 & 1 & $\begin{array}{l}1 \\
1\end{array}$ & $\begin{array}{l}r \\
r \\
r\end{array}$ & $\begin{array}{l}r \\
r \\
r \\
z\end{array}$ \\
\hline $\mathrm{Pf}_{12}$ & $\begin{array}{c}\mathbb{P}\left(1^{2}, 2, r^{2}, z\right) \\
r=2 n+1, n \geq 1 \\
w=(0,1,1, q, r)\end{array}$ & $\frac{1}{r}(2, q), \frac{1}{z}(1,1)$ & $\frac{2 r^{2}+5 r+1}{4 r^{2}-2 r}$ & 2 & 1 & $\begin{array}{l}1 \\
2\end{array}$ & $\begin{array}{l}q \\
r \\
r\end{array}$ & $\begin{array}{l}r \\
s \\
s \\
z\end{array}$ \\
\hline $\mathrm{Pf}_{13}$ & $\begin{array}{c}\mathbb{P}\left(1,2, r^{2}, z, m\right) \\
r=2 n+1, n \geq 1 \\
w=\frac{1}{2}(4-s, s-2 \\
s, 3 s-6,3 s-4)\end{array}$ & $\frac{1}{r}(2, q), \frac{1}{m}(r, z)$ & $\frac{3 r+1}{r(3 r-2)}$ & 1 & 1 & $\begin{array}{l}2 \\
r\end{array}$ & & $\begin{array}{l}s \\
z \\
2 r \\
m\end{array}$ \\
\hline $\mathrm{Pf}_{14}$ & $\begin{array}{c}\mathbb{P}\left(2, r^{2}, s, t, z\right) \\
r=2 n+1, n \geq 1 \\
w=\frac{1}{2}(1,3, z, z, 2 r+1)\end{array}$ & $\begin{array}{c}\frac{1}{t}(2, s), \frac{1}{z}(1,1) \\
3 \times \frac{1}{r}(2, q)\end{array}$ & $\frac{4 r+3}{r(r+2)(2 r-1)}$ & 0 & 2 & $\begin{array}{l}r \\
s\end{array}$ & & $\begin{array}{c}s \\
t \\
2 r \\
2 r\end{array}$ \\
\hline $\mathrm{Pf}_{21}$ & $\begin{array}{c}\mathbb{P}\left(1^{2}, 2,3, r, s\right) \\
r=3 n, n \geq 2 \\
w=(0,1,1,2, q)\end{array}$ & $\begin{array}{c}\frac{1}{3}(1,1), \frac{1}{r}(1,1), \\
\frac{1}{s}(3, r)\end{array}$ & $\frac{4\left(2 r^{2}+4 r+3\right)}{3 r(r+1)}$ & 2 & 1 & $\begin{array}{l}1 \\
2\end{array}$ & $\begin{array}{l}2 \\
3 \\
3\end{array}$ & $\begin{array}{l}q \\
r \\
r \\
s\end{array}$ \\
\hline $\mathrm{Pf}_{22}$ & $\begin{array}{l}\text { rest same as } \mathrm{Pf}_{21} \\
r=3 n+1, n \geq 2\end{array}$ & $\frac{1}{r}(1,1), \frac{1}{s}(3, r)$ & Same as $\mathrm{Pf}_{21}$ & 2 & San & ne & asP & $\mathrm{f}_{21}$ \\
\hline
\end{tabular}


Table 1 continued from previous page

\begin{tabular}{|c|c|c|c|c|c|c|c|c|}
\hline $\mathrm{Pf}_{23}$ & $\begin{array}{c}\mathbb{P}(1,3, r, s, t, u) \\
r=3 n+2, n \geq 2 \\
w=(0,1,2, r, s)\end{array}$ & $\begin{array}{c}\frac{1}{3}(1,1), \\
\frac{1}{r}(1,1), \frac{1}{u}(3, t)\end{array}$ & $\frac{8 r+36}{3 r^{2}+9 r}$ & 1 & 1 & $\begin{array}{l}2 \\
3\end{array}$ & $\begin{array}{l}r \\
s \\
t\end{array}$ & $\begin{array}{l}s \\
t \\
u \\
v\end{array}$ \\
\hline $\mathrm{Pf}_{24}$ & $\begin{array}{c}\mathbb{P}\left(3, r, s^{2}, t^{2}\right) \\
r=3 n, n \geq 2 \\
w=\frac{1}{2}(q, s, s, u, u)\end{array}$ & $\begin{array}{c}\frac{1}{3}(1,1), \frac{1}{r}(1,1), \\
\frac{1}{s}(3, r), 2 \times \frac{1}{t}(3, s)\end{array}$ & $\frac{4(5 r+6)}{3 r\left(r^{2}+3 r+2\right)}$ & 0 & $r$ & $\begin{array}{l}r \\
s\end{array}$ & $\begin{array}{l}s \\
t \\
t\end{array}$ & $\begin{array}{l}s \\
t \\
t \\
u\end{array}$ \\
\hline
\end{tabular}

The first model in the above table has already been discussed in [CP17] where its description as a toric variety has been provided.

In 44 we prove the existence, wellformedness and quasismoothness of some codimension 4 biregular models of log Del Pezzo surfaces with rigid singularities. They can be realized as regular pullbacks from $\mathbb{P}^{2} \times \mathbb{P}^{2}$ format which we denote by w $\mathcal{P}$. We recall the definition of weighted $\mathbb{P}^{2} \times \mathbb{P}^{2}$ and a formula for its Hilbert series from [Sze05]. We use the graded free resolution information from its Hilbert series to give a formula to compute the anti canonical degree $-K^{2}$ of $\log$ Del Pezzo surfaces in a given w $\mathcal{P}$ variety. In total there are four biregular models of index 1 . The case of index 2 models is quite special so we treat it separately.

Theorem 1.2. There are 4 biregular models of log Del Pezzo surfaces of Fano index 1 with baskets of rigid singularities in Table 0 such that a general member of each family in each model is wellformed and quasismooth. Their equations are described by $2 \times 2$ minors of the order 3 matrix of homogenous forms giving the embedding of each family $\mathcal{X} \hookrightarrow \mathbb{P}^{6}(a, \ldots, g)$. At least, one of these biregular models does not admit a $\mathbb{Q}$-Gorenstein deformation to a toric variety.

Table 2: biregular Log Del Pezzo Models in $\mathbb{P}^{2} \times \mathbb{P}^{2}$ format where $q=r-1, s=r+1, t=r+2, z=2 r-1$.

\begin{tabular}{|c|c|c|c|c|c|}
\hline Model & WPS \& Para & $\mathcal{B}$ & $-K_{X}^{2}$ & $h^{0}(-K)$ & Weight Matrix \\
\hline $\mathbf{P}_{11}$ & $\begin{array}{c}\mathbb{P}\left(1^{4}, r^{2}, z\right) \\
r \geq 2 \\
w=(0,0, q ; 1,1, r)\end{array}$ & $\frac{1}{z}(1,1)$ & $\frac{4 r+2}{1-2 r}$ & 4 & $\begin{array}{lll}1 & 1 & r \\
1 & 1 & r \\
r & r & z\end{array}$ \\
\hline $\mathbf{P}_{12}$ & $\begin{array}{c}\mathbb{P}\left(1,2, r^{2}, s, t, z\right) \\
r=2 n+1 n \geq 1 \\
w=(0,1, q ; 1, r, s)\end{array}$ & $\frac{1}{r}(2, q), \frac{1}{t}(2, s), \frac{1}{z}(1,1)$ & $\frac{2 r^{2}+7 r+1}{r(r+2)(2 r-1)}$ & 1 & $\begin{array}{ccc}1 & 2 & r \\
r & s & z \\
s & t & 2 r\end{array}$ \\
\hline
\end{tabular}


Table 2 continued from previous page

\begin{tabular}{|c|c|c|c|c|c|c|c|}
\hline $\mathbf{P}_{13}$ & $\begin{array}{c}\mathbb{P}\left(1,2^{2}, 3, r^{2}, z\right) \\
r=2 n+1, n \geq 1 \\
w=(0,1, q ; 1,2, r)\end{array}$ & $\begin{array}{l}\frac{1}{3}(1,1), \frac{1}{z}(1,1) \\
2 \times \frac{1}{r}(2, q)\end{array}$ & $\frac{(2 r+3)}{3 r(2 r-1)}$ & 1 & $\begin{array}{l}1 \\
2 \\
r\end{array}$ & $\begin{array}{l}2 \\
3 \\
s\end{array}$ & $\begin{array}{l}r \\
s \\
z\end{array}$ \\
\hline $\mathbf{P}_{14}$ & $\begin{array}{c}\mathbb{P}\left(2,3, r^{2}, s, t, z\right) \\
r=6 n-1, n \geq 1 \\
w=(0,1, q ; 2, r, s)\end{array}$ & $\begin{array}{c}\frac{1}{3}(1,1), 3 \times \frac{1}{r}(2, q) \\
\frac{1}{t}(3, r), \frac{1}{z}(1,1)\end{array}$ & $\frac{(r+1)(2 r+9)}{3 r(r+2)(2 r-1)}$ & 0 & $\begin{array}{l}2 \\
r \\
s\end{array}$ & $\begin{array}{l}3 \\
s \\
t\end{array}$ & $\begin{array}{c}s \\
z \\
2 r\end{array}$ \\
\hline
\end{tabular}

The first model has been discussed in [CP17] and its toric description has also been provided by embedding it in a singular toric variety. In the case of Fano index 2 we get two biregular models which are indexed by 2 parameters giving rise to an infinite series of one parameter biregular models in each case. More strikingly up to adjunction number 68 we do not get a single quasismooth family with rigid singularities which is not in one of these bi-parameterized models.

Theorem 1.3. Let $(r, y) \in \mathbb{N} \times \mathbb{N}$ be a pair of positive integers having one the following types:

$$
\begin{aligned}
& \text { - } r=3 m, y=3 n, m, n \geq 2 \\
& \text { - } r=3 m, y=3 n+1, m, n \geq 2,
\end{aligned}
$$

Then for each choice of input parameter $w=(0,1, q ; 1,2, y)$ we get a family of wellformed and quasismooth log Del Pezzo surfaces

$$
\mathcal{X} \hookrightarrow \mathbb{P}(1,2,3, r, s, y, z)
$$

with the weight matrix

$$
\left(\begin{array}{ccc}
1 & 2 & r \\
2 & 3 & s \\
y & z & w
\end{array}\right)
$$

where $q=r-1, s=r+1, z=y+1$ and $w=q+y$. The degree of the canonical divisor class in terms of parameters $r$ and $y$ is given by

$$
-K_{X}^{2}=\frac{4\left(r^{2}(2 y+3)+r\left(2 y^{2}+4 y+3\right)+3 y(y+1)\right)}{3 r y(r+1)(y+1)} .
$$

The basket of orbifold points on each family in both cases is given as follows.

\begin{tabular}{c|c}
\hline Model & Basket \\
\cline { 2 - 2 } $\mathbf{P}_{21}$ & $\frac{1}{3}(1,1), \frac{1}{r}(1,1), \frac{1}{s}(3, r), \frac{1}{y}(1,1), \frac{1}{z}(3, y)$ \\
$\mathbf{P}_{22}$ & $\frac{1}{r}(1,1), \frac{1}{s}(3, r), \frac{1}{y}(1,1), \frac{1}{z}(3, y)$ \\
\hline
\end{tabular}


It is important to mention that the computer search gives another model with $r=3 m+1$ and $y=3 n$ but due to symmetry of the weight matrix along the diagonal it is isomorphic to $\mathbf{P}_{22}$.

In $\$ 5$ we gave a summary of computational results obtained by using the computer search in each of these two formats. The summary consists of a number of candidate families returned by computer, how many of those contain only rigid singularities and how many of them are quasismooth. We finish by presenting the complete list of sporadic cases of families of wellformed and quasismooth log Del Pezzo surfaces appearing in these formats, up to certain adjunction number.

Understanding Table 1 and $\mathbf{Q}$. The first column denotes the model name where $\operatorname{Pf}_{i j}\left(\mathbf{P}_{i j}\right)$ represents $j$-th model of index $i$. The second column contains the weights of the ambient weight projective space containing $X$ and two types of parameters: the first one tells us the form of the parameter $r$ and the second one gives the weights of the syzygy matrix appearing in the last column of the table. The 3rd column contains the basket of singularities on $X$. The column $-K^{2}$ represents the degree of the canonical class $-K_{X}$ of $X$ in terms of parameter $r$ and $h^{0}(-K)$ contains the first plurigenus of its Hilbert series. The last column contains the so called syzygy or weight matrix of $X$.

Acknowledgement. I wish to thank Alexander Kasprzyk for some enlightening discussions which made me think about this project. I am also thankful to Gavin Brown and Yuri Prokhorov for helpful comments. Thanks are also due to Nouman Zubair for setting up MAGMA on the HPC cluster of LUMS to run the computer calculations for this paper. Last but not least, I am grateful to an anonymous referee for helping me improve an earlier version of the paper a great deal. This research is supported by an Higher Education Commission (HEC)'s NRPU grant 5906/Punjab/NRPU/RD/HEC/2016 and a fellowship of the Alexander-von-Humboldt foundation.

\section{Preliminaries, Notation and General strategy of proofs}

2.1. Preliminaries. Let $\mu_{r}$ denote the cyclic group generated by a primitive $r$-th root of unity. Its acts on $\mathbb{A}_{x, y}^{2}$ by $x \mapsto \epsilon^{a} x, y \mapsto \epsilon^{b} y$. The quotient is called a cyclic quotient singularity or an orbifold point of type $\frac{1}{r}(a, b)$. It is called isolated if $r$ is relatively prime to $a$ and $b$. A singularity is called a T-singularity if it admits a $\mathbb{Q}$-Gorenstein smoothing. It is called an $R$-singularity it it is is rigid under any $\mathbb{Q}$-Gorenstein smoothing. We use the following characterization of a cyclic quotient singularity to be a $T$-singularity and $R$-singularity, appeared in CP17.

Definition 2.1. Given an arbitrary quotient singularity $Q=\frac{1}{r}(a, b)$, let $m=\operatorname{gcd}(a+b, r)$, $d=(a+b) / m$ and $k=r / m$. Then $Q$ can be written in the form $\frac{1}{m k}(1, m d-1)$ and if :

(i) $k \mid m$ then $Q$ is a $T$-singularity [KSB88;

(ii) $m<k$ then $Q$ is a $R$-singularity [AK14] .

An algebraic surface is $\mathbb{Q}$-Gorenstein if it is normal and the canonical divisor class is a $\mathbb{Q}$-ample Weil divisor. A $\mathbb{Q}$-Gorenstein algebraic surface $X$ is a del Pezzo surface if the anti-canonical divisor class $-K_{X}$ is ample. If $X$ has at worst only isolated quotient 
singularities then it is called orbifold or log Del Pezzo surface. The largest positive integer $I$ such that $-K_{X}=I \cdot D$ for some element $D$ in the divisor class group of $X$, is known as the Fano index of a log Del Pezzo surface $X$.

Definition 2.2. A biregular model of log Del Pezzo surfaces is an infinite series of families of $\log$ Del Pezzo surfaces satisfying the following conditions.

(i) There exist a family of $\log$ Del Pezzo surfaces for each value of the parameter $r(n)$ for all $n \in \mathbb{N}$.

(ii) Each family has the embedding $\mathcal{X} \hookrightarrow \mathbb{P}\left(w_{i}\right)$ such that at least one of the weights is $r$, and each weight $w_{i}$ and the degree of the canonical class $\left(-K_{\mathcal{X}}\right)^{2}$ are functions of $r$.

We may only use the word "model" and "biregular model" interchangeably if no confusion can arise. A biregular model is called wellformed and quasismooth model if a general member in each family is wellformed and quasismooth log Del Pezzo surface. A surface $X \subset \mathbb{P}\left(w_{i}\right)$ is quasismooth if the affine cone $\widetilde{X}$ over $X$ is smooth outside the vertex $\underline{0}$ and wellformed if at worst it contain the isolated orbifold points. We use the algorithmic approach of BKZ, Qur17a to search for the candidate biregular models which is primarily based on a theorem of Buckley, Reid and Zhou [BRZ13]. The theorem gives a decomposition of the Hilbert series $P(t)$ of a projectively Gorenstein orbifold $X$ with isolated orbifold points into a smooth and orbifold part. The Gorenstein assumption on a surface $X$ with $K_{X}=I \cdot D$ implies that $\frac{1}{r}(a, b)$ must satisfy

$$
a+b+I=0 \bmod r .
$$

Lemma 2.3. Let $X$ be a log Del Pezzo surface of Fano index $1 \leq I \leq 2$, then the orbifold point $Q=\frac{1}{r}(a, b)$ is a T-singularity if it is either $\frac{1}{2}(1,1)$ or $\frac{1}{4}(1,1)$. Otherwise, it is an $R$-singularity.

Proof. The proof follows from a straightforward application of 2.1. If $m=\operatorname{gcd}(a+b, r)$ then for $I=1$ there are no orbifold point of type $\frac{1}{2}(1,1)$ and $\frac{1}{4}(1,1)$ on $X$, due to (1). Otherwise, we have $a+b=r+1$ thus $m=1<k=r$. If the Fano index $I=2$ then if $r$ is odd then $m=1<k=r$. Otherwise, if $r$ is even then $m=2$ and $k=r / 2$. Now $k \mid m$ if $r=2,4$ otherwise $m<k$.

In our proofs and calculations we repeatedly use the following Lemma to compute the exact number of singular points on orbifold loci of our varieties.

Lemma 2.4. [IF00, Lemma 9.4] Let $X \subset \mathbb{P}\left(a_{0}, a_{1}\right)$ be a general hypersurface of degree $d$ with $\operatorname{gcd}\left(a_{0}, a_{1}\right)=1$. If $P_{0}$ and $P_{1}$ denote the coordinate points $(1,0)$ and $(0,1)$ respectively, then $X$ is a finite set such that $P_{i} \in X$ if $a_{j} \nmid d$ for any $j=0,1$ and it contains $\left\lfloor\frac{d}{a_{0} a_{1}}\right\rfloor$ further points.

\subsection{Notation.}

- We work over the field of complex numbers $\mathbb{C}$. We write $\mathcal{X}$ for a family of Del Pezzo surfaces and $X$ for its general member. 
- Any isolated orbifold point $\frac{1}{r}(a, b)$ can be written in the form $\frac{1}{r}\left(1, b^{\prime}\right)$ by using a different primitive generator of $\mu_{r}$, We use the latter form of the orbifold points in all tables and examples. We use the term quotient singularity and orbifold point interchangeably.

- All our orbifolds are projectively Gorenstein so each orbifold point of type $\frac{1}{r}(1, b)$ has a presentation which satisfies the condition (11).

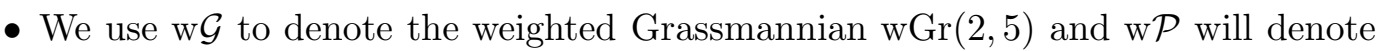
the ambient weighted $\mathbb{P}^{2} \times \mathbb{P}^{2}$ variety.

- $a, b, c, d, e, f$ and $g$ represent the variables on the ambient weight projective space containing a Del Pezzo surface $X$ whereas $m, r, s, t, u, v, y$, and $z$ denote the weights of the variables depending on the model. The subscripts will denote the degree of these variables in the proofs.

- The capital letters like $H_{d} s$ and $J_{d} s$ (or only $(d)$ ) denote homogeneous forms of degree $d$.

- We enclose the matrix of weights inside parentheses () and matrix of variables and homogeneous forms inside square brackets [] in the proofs. If we need to distinguish between two weights of same degree in the weight matrix then we distinguish them with subscript, e.g. for example if we have two weights of degree $z$ then we denote them by $z_{1}, z_{2}$ in the weight matrix.

2.3. General strategy of the proofs. For each model, and the sporadic examples of 95. the proofs are divided into the following steps.

2.3.1. Existence. The first part is to show the existence of such models. We show the existence of such models by constructing them as quasilinear sections of the given ambient key variety $\mathrm{w} \operatorname{Gr}(2,5)$ or $w\left(\mathbb{P}^{2} \times \mathbb{P}^{2}\right)$ by specifying the choice of input parameters and quasilinear sections. The most important part of the existence is to show that each family of Del Pezzo orbifolds $\mathcal{X}$ contains exactly those singular points which are suggested by the output in the computer search. A family may fail when it does not contain a suggested orbifold point, or sometimes when it contains 1-dimensional orbifold singularities.

2.3.2. Wellformedness and Quasismoothness. The wellformedness part is quite simple and follows straight away from the existence of models with right singularities in this case. If $X \subset \mathbb{P}\left(a_{0}, \cdots, a_{n}\right)$ is an orbifold Del Pezzo surface then the orbifold singularities on $X$ occur due to the singularities of $\mathbb{P}\left(a_{0}, \cdots, a_{n}\right)$. We start by computing the dimensions of each orbifold locus of $\mathbb{P}\left(w_{i}\right)$ restricted to $X$ which answers the question of wellformedness. Since we are on a surface, $X$ is wellformed if and only if, at worst, it intersects in finite number of points with the singular strata of $\mathbb{P}\left(w_{i}\right)$.

The quasismoothness needs some detailed and careful analysis of two different types of loci. One comes from the singularities of ambient weighted projective space. The second one may appear due to the base loci of the successive linear systems of the intersecting weighted homogeneous forms. Outside of these loci, a general member in each family of these models remain quasismooth due to the following version of Bertini's theorem. 
Theorem 2.5 (Bertini). If a hypersurface $X \subset \mathbb{P}\left(a_{0}, \ldots, a_{n}\right)$ is a general element of a linear system $L=|\mathcal{O}(d)|$, then the singularities (non-quasismooth points) of $X$ may only occur on the reduced part of the base locus of $L$.

If $p$ is an orbifold point of type $\frac{1}{r}(a, b)$ then it is either a coordinate point or lies on the strata of dimension $\geq 1$. Usually $p$ is a coordinate point and we show that there exist $l$ weighted homogenous equations of the form

$$
F_{k}:=x_{i}^{m} x_{k}+\cdots
$$

in the ideal of $X$ where $l$ is the codimension of $X, x_{i}$ is the $i$-th coordinate point with weight $a_{i}:=r$ and the remaining two variables have weights $a$ and $b$ modulo $r$. These $l$ equations are called tangent polynomials [BZ10]. We call the variables $x_{k}$ the tangent variables and rest of the variables local variables near the point $p$.

Since we consider the description of these models of log Del Pezzo surfaces as complete intersections in some ambient $\mathrm{w} \operatorname{Gr}(2,5)$ or $\mathrm{w}\left(\mathbb{P}^{2} \times \mathbb{P}^{2}\right)$, i.e. a general memeber $X$ is of the form

$$
X=(\mathrm{w} \mathcal{G} \text { or } \mathrm{w} \mathcal{F}) \cap\left(\cap\left(d_{i}\right)\right) \subset \mathbb{P}\left(w_{i}\right) .
$$

There is a base locus of the linear systems $\left(\left|\mathcal{O}\left(d_{i}\right)\right|\right)$ of each form of degree $d_{i}$ where $X$ may have singularities by Theorem 2.5. To prove that $X$ is quasismoothness on the base locus, in few cases we use a purely theoretical arguments and mostly, a combination of theoretical and computational evidence. A theoretical arguments works well if $X$ intersect with the base loci in finite number of points. Otherwise the base loci is very complicated and it becomes very difficult to show the quasismoothness theoretically. In such cases, we show that the base locus remains geometrically does not change for each value of the parameter $r$. We give a detailed proof in one of the cases, i.e. in section 3.2 .2 and rest of the cases are similar. Thus it suffices to show that $X$ is quasismooth for first few values of $r$, to establish quasismoothness for a given model. For small values of $r$, we show the quasismoothness by using the computer algebra system MAGMA by writing down its equations over the rational numbers. To provide further evidence, we verify it for as large values of $r$ as computationally feasible by computer algebra. We write down the largest value of parameter which we checked by computer algebra, in each case separately in the proofs.

2.3.3. Non existence of deformations to a toric variety. Each family in our models of orbifold Del Pezzo surfaces is locally $\mathbb{Q}$-Gorenstein rigid. From $\left[\mathrm{ACC}^{+} 16\right]$, we know that $h^{0}(-K)$ (more generally all plurigenera $h^{0}(-m K)$ ) is invariant under $\mathbb{Q}$-Gorenstein deformations. Now if $X_{\Sigma}$ is a toric Fano variety then $h^{0}(-K)>0$ since the origin is always contained in the corresponding Fano polytope. Thus if each family in our model has $h^{0}(-K)=0$ then the we say that the model does not admit a $\mathbb{Q}$-Gorenstein deformation to a toric variety. Such deformation families are more interesting in a sense that they can not be constructed using toric methods. In fact two of our models do admit $\mathbb{Q}$-Gorenstein deformation to toric varieties, these appeared in [CP17]. But we do not treat this question in this paper for all our orbifold models. 


\section{PfaffiAn MODElS}

3.1. Generalities on $\mathrm{w} \operatorname{Gr}(2,5)$. This part mainly consists of the selected material from CR02 where the detailed treatment of the subject can be found. In the rest of this section we denote it by $\mathrm{w} \mathcal{G}$.

Definition 3.1. Consider a 5 tuple of all integers or half integers $w:=\left(w_{1}, \cdots, w_{5}\right)$ such that

$$
w_{i}+w_{j}>0,1 \leq i<j \leq 5
$$

Then the w $\mathcal{G}$ is the quotient of punctured affine cone $\widehat{\operatorname{Gr} \backslash\{0\}}$ by $\mathbb{C}^{\times}$:

$$
\lambda: x_{i j} \mapsto \lambda^{w_{i}+w_{j}} x_{i j}
$$

where $x_{i j}$ are Plücker coordinates of the embedding $\operatorname{Gr}(2,5) \hookrightarrow \mathbb{P}\left(\bigwedge^{2} \mathbb{C}^{5}\right)$. Thus we get the embedding

$$
{ }_{\mathrm{w}} \mathcal{G} \hookrightarrow \mathbb{P}\left(\left\{w_{i}+w_{j}: 1 \leq i<j \leq 5\right\}\right),
$$

which is defined by the 5 maximal Pfaffians of $5 \times 5$ skew symmetric.

We refer to this skew symmetric matrix as weight matrix and usually present by only writing down the upper triangular part,

$$
\left(\begin{array}{llll}
w_{12} & w_{13} & w_{14} & w_{15} \\
& w_{23} & w_{24} & w_{25} \\
& & w_{34} & w_{35} \\
& & & w_{45}
\end{array}\right),
$$

where $w_{i j}=w_{i}+w_{j}$. The Hilbert series of $\mathrm{w} \mathcal{G}$ is given by

$$
P_{\mathrm{w} \mathcal{G}}=\frac{1-\sum_{i=1}^{5} t^{d-w_{i}}+\sum_{i=1}^{5} t^{d+w_{i}}-t^{2 d}}{\prod_{i, j}\left(1-t^{w_{i}+w_{j}}\right)}
$$

where $d=\sum w_{i}$. If $\mathrm{w} \mathcal{G}$ is wellformed, the orbifold canonical canonical class is

$$
K_{\mathrm{w}} \mathcal{G}=\mathcal{O}\left(2 d-\sum_{i, j} w_{i}+w_{j}\right)=\mathcal{O}(-2 d) .
$$

The degree of weighted Grassmannian w $\mathcal{G}$ is

$$
\operatorname{deg} \mathrm{w} \mathcal{G}=\frac{\left(\begin{array}{c}
2 d \\
3
\end{array}\right)-\sum_{i=1}^{5}\left(\begin{array}{c}
d+w_{i} \\
3
\end{array}\right)+\sum_{i=1}^{5}\left(\begin{array}{c}
d-w_{i} \\
3
\end{array}\right)}{\prod\left(w_{i}+w_{j}\right)}
$$

Then obviously if $X=\mathrm{w} \mathcal{G} \cap\left(\cap_{i=1}^{4}\left(f_{i}\right)\right)$ is complete intersection Del Pezzo orbifold of index $I$ then

$$
-K_{X}^{2}=I^{2} \prod_{i=1}^{4} \operatorname{deg}\left(f_{i}\right) \operatorname{deg}(\mathrm{w} \mathcal{G})
$$


The degree of each model has been computed using the above formula by using the computer algebra software Mathematica.

3.2. Proof of Theorem 1.1. We first give the proof of existence of each model with right invariants and singularities. Then we prove the quasismoothness of the each model. Here we are taking the point of view appeared Qur17a, QS12, Qur17b, i.e. to consider each $X$ as weighted complete intersection of some $\mathrm{w} \mathcal{G}$. Indeed, it is a a special case of being consider them as regular pullbacks of some key variety, like in [BKZ]. We write the proof for a general member $X$ of a family $\mathcal{X} \subset \mathbb{P}(a, b, c, d, e, f)$ of orbifold Del Pezzo surfaces. In the course of the proof our parameter is $r$ and the rest of the weights in terms of $r$ are

$$
\begin{array}{llll}
q=r-1, & s=r+1, & t=r+2, & u=r+3, \\
v=2 r+1, & y=2 r-2, & z=2 r-1, & m=3 r-2 .
\end{array}
$$

3.2.1. Pfaffian Model 11. This is the simplest of Pfaffian models and quite straight forward to prove each family is quasi sooth. The parameter is $r=n, n \in \mathbb{N}$ and if we choose an input parameter $w=\frac{1}{2}(1,1,1, z, z)$ we get the embedding of 6 dimensional orbifold

$$
{ }_{\mathrm{w}} \mathcal{G} \hookrightarrow \mathbb{P}\left(1^{3}, r^{6}, z\right) \text { with the orbifold canonical class } K_{\mathrm{w}} \mathcal{G}=\mathcal{O}(-4 r-1) .
$$

Then if we take the complete intersection of $\mathrm{w} \mathcal{G}$ with 4 forms of degree $r$ then

$$
X:=\mathrm{w} \mathcal{G} \cap(r)^{4} \hookrightarrow \mathbb{P}\left(1^{3}, r^{2}, z\right)=\mathbb{P}(a, b, c, d, e, f)
$$

is a Del Pezzo surfaces of index 1.

Quasismoothness: $X$ has two non-trivial orbifold strata, one is of weight $z$ and the other of weight $r$. The weight $z$ locus is just a point which obviously lies on $X$. The variables $a, b, c$ serve as tangent variables and $d, e$ as local variables near to $f \neq 0$. Thus it is a point of type $\frac{1}{z}(r, r)=\frac{1}{z}(1,1)$. On the other hand the weight $r$ locus is a copy of the Segre 3 -fold $\mathbb{P}^{1} \times \mathbb{P}^{2}$ in w $\mathcal{G}$ which does not intersect $X$.

The base locus of linear system $|\mathcal{O}(r)|$ consists of just a coordinate point of weight $z$ :

$$
\mathcal{B} s(|\mathcal{O}(r)|)=(0,0,0,0,0,1),
$$

which is quasismooth. Thus the each member in this models is a wellformed and quasismooth family of log Del Pezzo surfaces.

3.2.2. Pfaffian model 12. The paramter has the form $r=2 n+1, n \in \mathbb{N}$ and for an input parameter $w=(0,1,1, q, r)$, we get

$$
\mathrm{w} \mathcal{G} \hookrightarrow \mathbb{P}\left(1^{2}, 2, q, r^{3}, s^{2}, z\right) \text { with } K_{\mathrm{w} \mathcal{G}}=\mathcal{O}(-2(2 r+1)) .
$$

If we take the complete intersection of $\mathrm{w} \mathcal{G}$ with two forms of degree $s$ and one form each of degree $r$ and $q$ then

$$
X:={ }_{\mathrm{w}} \mathcal{G} \cap(s)^{2} \cap(q) \cap(r) \hookrightarrow \mathbb{P}\left(1^{2}, 2, r^{2}, z\right)=\mathbb{P}(a, b, c, d, e, f)
$$


is a log Del Pezzo surfaces of Fano index 1 . The equations are given by the $4 \times 4$ Pfaffians of skew symmetric matrix

$$
\left[\begin{array}{cccc}
a_{1} & b_{1} & H_{q} & d_{r_{1}} \\
& c_{2} & H_{r_{2}} & H_{s_{1}} \\
& & e_{r_{3}} & J_{s_{2}} \\
& & & f_{z}
\end{array}\right],
$$

where $H$ and $J$ denote the general form of degree and the subscripts denotes the weights of variables and general forms.

Quasismoothness: Now $X$ has 3 different orbifold loci, having weight $z, r$ and 2 . The weight $z$ locus is again just a point which obviously lies on $X$. It is obviously a point of type $\frac{1}{z}(1,1)$.

The weight $r$ locus can be taken as a coordinate point $(0,0,0,0,1,0)$ of the variable $e$. Let

$$
H_{s}=b e+\cdots
$$

then $a, b$ and $d$ are tangent variables. Then we get $c$ and $f$ are local variables near this point which gives an orbifold point of type $\frac{1}{r}(2, q)$.

The weight 2 locus on $X$ is $V\left(c f, c H_{q}\right) \subset \mathbb{P}(2, z)$ which is an empty set.

Now we analyze the base loci of each linear system of weighted homogenous forms. We start with

$$
X_{1}=\mathrm{w} \mathcal{G} \cap(s)^{2} \subset \mathbb{P}\left(1^{2}, 2, q, r^{3}, z\right) ;
$$

the intersection of ${ } \mathcal{G}$ with two forms of weight $s$. Then the base locus of the linear system of degree $s$ restricted to $X_{1}$ is

$$
\mathcal{B} s(|\mathcal{O}(s)|) \cap X_{1}=\mathbb{P}\left[q, r_{1}, r_{2}, z\right] \cup \mathbb{P}\left[q, r_{3}, z\right] .
$$

Then $X_{1}$ is quasismooth away from this locus. If we take $X_{2}=X_{1} \cap(q)$ and then we have

$$
\mathcal{B} s(|\mathcal{O}(q)|) \cap X_{2}=\mathbb{P}\left[r_{1}, r_{2}, z\right] \cup \mathbb{P}\left[r_{3}, z\right] .
$$

We do not get any new base locus in this case and $X_{2}$ is quasismooth away from this locus. At the end, we get $X=X_{2} \cap\left(r_{2}\right)$ which intersects with the base locus of $\left|\mathcal{O}\left(r_{2}\right)\right|$ in two coordinate points of $\mathbb{P}[2, z]$ which are manifestly quasismooth. Moreover, taking last two intersections reduces $\mathcal{B} s(|\mathcal{O}(q)|)$ to $\mathbb{P}\left[r_{2}, z\right] \cup \mathbb{P}\left[r_{3}, z\right]$. Thus $X$ is quasismooth outside this locus. The loucs geometrically does not change for all values of the parameter and we use the computer algebra to prove the quasismoothness of this model. The following MaGma function shows the quasismoothness for any value of the parameter $r$.

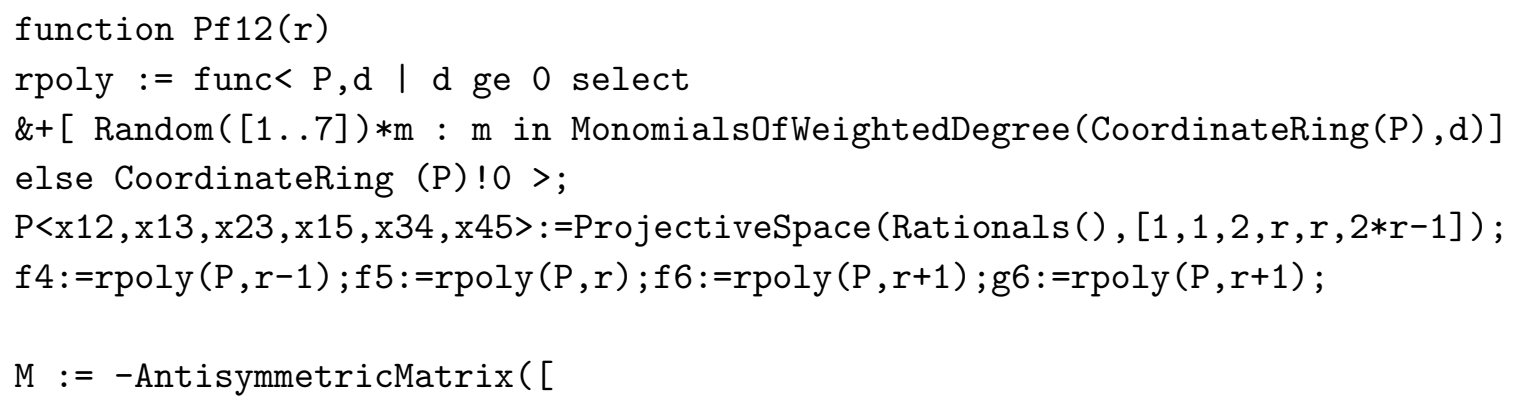


$\mathrm{x} 12$,

$\mathrm{x} 13, \mathrm{x} 23$,

$f 4, f 5, x 34$,

$\mathrm{x} 15, \mathrm{f} 6, \mathrm{~g} 6, \mathrm{x} 45])$;

$X:=$ Scheme (P,Pfaffians $(M, 4))$;

SX := JacobianSubrankScheme $(X)$;

SXred := ReducedSubscheme(SX);

D: =Dimension(SXred);

return $\mathrm{D}$;

end function;

In this model, we checked the quasismoothness for $3 \leq r \leq 49$.

3.2.3. Pfaffian model 13. The parameter has the form $r=2 n+1, n \in \mathbb{N}$ and if we choose an input parameter

$$
w=\frac{1}{2}(4-s, s-2, s, 3 s-6,3 s-4)
$$

we get an embedding of

$$
\mathrm{w} \mathcal{G} \hookrightarrow \mathbb{P}\left(1,2, r^{2}, s, y, z^{2}, 2 r, m\right) \text { with } K_{\mathrm{w}} \mathcal{G}=\mathcal{O}(-(7 r-1)) .
$$

If we take the complete intersection of $\mathrm{w} \mathcal{G}$ with four forms having degrees $z, 2 r, y$ and $r$, we get a log Del Pezzo surface

$$
X:=\mathrm{w} \mathcal{G} \cap(z) \cap(2 r) \cap(y) \cap(s) \hookrightarrow \mathbb{P}\left(1,2, r^{2}, z, m\right)=\mathbb{P}(a, b, c, d, e, f) .
$$

The equations can be described by the maximal Pfaffians of the skew symmetric matrix

$$
\left[\begin{array}{cccc}
a_{1} & b_{2} & c_{r} & H_{s} \\
& d_{r} & H_{y} & H_{z} \\
& & e_{z} & H_{2 r} \\
& & & f_{m}
\end{array}\right] .
$$

Quasismoothness: We show that $X$ has only four distinct orbifold loci with weights $m, z, r$ and 2 .

The weight $m$ locus is just the coordinate point of the variable $f$ which lies on $X$. The variables $a, b$ and $d$ can be removed by using the implicit function theorem near this point and $c, e$ are local variables near this point. Thus we get an orbifold point of type $\frac{1}{m}(r, z)$. This indeed represents a point as $m$ is relatively prime to both $r$ and $z$.

The weight $z$ locus is just an empty set as we have a term $e^{2}$ in one of the defining equations of $X$. The equations of $X$ restricted to weight $r$ variables is given by

$$
V\left(c H_{2 r}, c d\right) \subset \mathbb{P}(c, d)
$$

which is manifestly the coordinate point of variable $d$ on $X$. If $H_{s}=a d+\cdots$ then $a, c$ and $f$ are tangent variables and we get an orbifold point of type $\frac{1}{r}(2, q)$ on $X$.

The weight 2 locus consists of intersection of $X$ with $\mathbb{P}(2, m)$ as $m$ is even. In the equations 
bf and $b H_{y}$; the variable $f$ does not appear in $H_{y}$ due to the reason of degree so it can be at most a coordinate point of variable $m$ which we already considered earlier. Thus we do not get any $\frac{1}{2}$ type of singular points on $X$.

In this case we can also show that the base locus geomtrically remains constant for any value of $r$, like in 3.2.2. Thus we used a computer algebra calculation and verified the quasismoothness for all $3 \leq r \leq 199$.

3.2.4. Pfaffian model 14. The paramter has the form $r=2 n+1, n \in \mathbb{N}$ and for an input parameter $w=\frac{1}{2}(1,3, z, z, 2 r+1)$ we get an embedding of

$$
{ }_{\mathrm{w}} \mathcal{G} \hookrightarrow \mathbb{P}\left(2, r^{2}, s^{3}, t, z,(2 r)^{2}\right) \text { with } K_{\mathrm{w} \mathcal{G}}=\mathcal{O}(-3(2 r+1)) .
$$

Then a Del Pezzo surface $X$ of index 1 is a complete intersection of w $\mathcal{G}$ with two forms each of degree $2 r$ and $s$.

$$
X:=\mathrm{w} \mathcal{G} \cap(2 r)^{2} \cap(s)^{2} \hookrightarrow \mathbb{P}\left(2, r^{2}, s, t, z\right)=\mathbb{P}(a, b, c, d, e, f) .
$$

The equations can be described by the maximal Pfaffians of the skew symmetric matrix

$$
\left[\begin{array}{cccc}
a_{2} & b_{r} & c_{r} & d_{s} \\
& H_{s} & J_{s} & e_{t} \\
& & f_{z} & H_{2 r} \\
& & & J_{2 r}
\end{array}\right] .
$$

Quasismoothness: We show that each $X$ has only three types of singularities with weights $r, t$ and $z$ and no other orbifold singularities.

The weight $z$ locus is just a coordinate point and $a, d$ and $e$ serve as tangent variables. Thus we get $b$ and $c$ as local variables near this point and we get an orbifold point of type $\frac{1}{z}(1,1)$.

The weight $t$ locus is again just a coordinate point. In this case, $b, c$ and $f$ are tangent variables so it is an orbifold point of type $\frac{1}{t}(2, s)$. Since $t$ is odd, this is only isolated singular point.

$X$ restricted to weight $s$ locus is an empty set as we have a pure power of $d$ appearing in the equations of $X$. The equations of $X$ restricted to weight $r$ variables is manifestly a cubic in $\mathbb{P}^{1}$ given by

$$
V\left(b J_{2 r}-c H_{2 r}\right) \subset \mathbb{P}(b, c) .
$$

If $H_{2 r}=c b+\cdots$, and $J_{2 r}=b c+\cdots$, then on each affine piece we can easily show that the local variables are of weight 2 and $z$ which gives us the 3 points of type $\frac{1}{r}(2, q)$ on $X$. At the end the weight 2 locus, $X \cap \mathbb{P}(2, s)$ as $s$ is even, does not intersect $X$.

Thus each $X$ contains correct type of orbifold singularities.

In this case we used computer algebra to verify the quasismoothness for $3 \leq r \leq 199,1161 \leq$ $r \leq 1199$ and $11161 \leq r \leq 11199$. Three different ranges were chosen to further assert the verification of quasismoothness. 
Index 2 models. The proof for index 2 cases are very similar to index 1 models. Therefore, we will give short summary of the quasismoothness on the orbifold locus in a tabular form to illuminate all the properties of the proof. We list the details of each orbifold loci in a small tabular form by writing down the tangent variables and the local variables in the neighbourhood of the corresponding open affine patches. Moreover, we also write the conditions needed on the intersecting weighted homogeneous forms to find all the tangent variables in each case. We will denote the weight of the singular strata under consideration with $P_{\text {orb}}$.

3.2.5. Pfaffian Model 21. The paramter has the form $r=3 n, n \in \mathbb{N}$ and for the input parameter $w=(0,1,1,2, q)$ we get the ambient weighted projective variety

$$
\mathrm{w} \mathcal{G} \hookrightarrow \mathbb{P}\left(1^{2}, 2^{2}, 3^{2}, q, r^{2}, s\right) \text { with } K_{\mathrm{w} \mathcal{G}}=\mathcal{O}(-2(r+3)) .
$$

Then the complete intersection of $\mathrm{w} \mathcal{G}$ with four forms of degree $r, q, 3$ and 2 is a log Del Pezzo surface

$$
X:=\mathrm{w} \mathcal{G} \cap(r) \cap(q) \cap(3) \cap(2) \hookrightarrow \mathbb{P}\left(1^{2}, 2,3, r, s\right)=\mathbb{P}(a, b, c, d, e, f)
$$

of index 2. The equations are given by the maximal Pfaffians

$$
\left[\begin{array}{cccc}
a_{1} & b_{1} & c_{2} & H_{q} \\
& H_{2} & d_{3} & e_{r} \\
& & H_{3} & H_{r} \\
& & & f_{s}
\end{array}\right]
$$

Quasismoothness: We summarise the details of the orbifold loci as follows.

\begin{tabular}{cccl}
$P_{\text {orb }}$ & $X \cap P_{\text {orb }}$ & Tangent $\mid$ local variables & Conditions on forms \\
\hline$s$ & coordinate pt $f_{s}$ & $a, b, c \mid d, e$ & $H_{2}=c+\cdots$ \\
$r$ & coordinate pt $e_{r}$ & $b, c, d \mid a, f$ & $H_{3}=d+\cdots$ \\
3 & coordinate pt- $d_{3}$ & $b, e, c \mid a, f$ & $H_{q}=d^{n} c+\cdots$
\end{tabular}

Since $r$ is a multiple of 3 , the weight 3 orbifold locus is

$$
V\left(d H_{r}-e H_{3}\right) \subset \mathbb{P}[3, r] .
$$

This is manifestly 2 points by using Lemma 2.4. The one new point is the coordinate point $d$ of weight 3 . The other one already appeared as weight $r$ orbifold point. The weight 2 locus is $\mathbb{P}[2, r]$ if $r$ is even and and $\mathbb{P}[2, s]$ otherwise. In both cases it does not intersect $X$ as $H_{2}=c+\cdots$.

Thus $X$ is wellformed and quasismooth on the orbifold locus. In this model, we use the computer algebra to checked that $X$ is quasismooth. We verify it for $6 \leq r \leq 69$ that it is quasismooth. Since the base locus remains the same for all $n$, we conclude that $X$ is quasismooth for all values of $n$. 
3.2.6. Pfaffian Model 22. This case is exactly similar to the 3.2.5 albeit our parameter is $r=3 n+1$. The proof of quasismoothness is only different at weight 3 and 2 orbifold loci. The weight 3 orbifold locus is just the coordinate point of variable $d$, which does not lie on $X$ as $H_{q}=d^{n}+\cdots$. Similarly the weight 2 locus is $\mathbb{P}[2, r]$ if $r$ is odd and $\mathbb{P}[2, s]$ otherwise. In both cases it does not intersect $X$.

Thus $X$ is wellformed and quasismooth on the orbifold locus and has the right type of singularities. Just like the last case, we used the computer algebra to verify that $X$ is quasismooth which gives a $7 \leq r \leq 70$. Since the base loci remains the same for all $r$, we conclude that this model is quasismooth for all values of $r$.

3.2.7. Pfaffian Model 23. The paramter has the form $r=3 n+2, n \in \mathbb{N}$ and for a choice of input parameter $w=(0,1,1, r, s)$ we get the ambient weighted projective variety

$$
{ }_{\mathrm{w}} \mathcal{G} \hookrightarrow \mathbb{P}\left(1,2,3, r, s^{2}, t^{2}, u, v\right) \text { with } K_{\mathrm{w}} \mathcal{G}=\mathcal{O}(-4(r+2)) .
$$

We take the complete intersection of $\mathrm{w} \mathcal{G}$ with four forms of degrees $v, t, s$ and 2 , to get a log Del Pezzo surface

$$
X:=\mathrm{w} \mathcal{G} \cap(v) \cap(t) \cap(s) \cap(2) \hookrightarrow \mathbb{P}(1,3, r, s, t, u)=\mathbb{P}(a, b, c, d, e, f)
$$

of index 2. The equations are given by the maximal Pfaffians of the skew symmetric matrix

$$
\left[\begin{array}{cccc}
a_{1} & H_{2} & b_{r} & H_{s} \\
& c_{3} & d_{s} & H_{t} \\
& & e_{t} & f_{u} \\
& & & H_{v}
\end{array}\right] .
$$

Quasismoothness: The details of weight $s$ and weight $r$ orbifold point is given in the following small table.

$$
\begin{array}{cccl}
P_{\text {orb }} & X \cap P_{\text {orb }} & \text { Tangent|local variables } & \text { Conditions } \\
u & \text { coordinate pt } f_{u} & a, b, d \mid c, e & \\
r & \text { coordinate pt } b_{r} & c, e, f \mid a, d & H_{t}=e+\cdots \\
3 & \text { coordinate pt } c_{3} & b, d, f \mid a, e & H_{v}=c^{n} f+\cdots
\end{array}
$$

The orbifold strata of weight $t$ and $s$ misses $X$ as we have the pure powers of $e$ and $d$ in the equations of $X$ if

$$
H_{t}=e+\cdots \text { and } H_{s}=d+\cdots .
$$

The weight 3 locus is $X \cap \mathbb{P}[3, s]$; explicitly

$$
V\left(d H_{s}, c d\right) \subset \mathbb{P}[c, d]
$$

which is the coordinate point of the variable $c$. Even though there is no variable of weight 2 in the ambient $w \mathbb{P}$ but

$$
\operatorname{GCD}(r, t)=2 \text {; if } r \text { is even and } \operatorname{GCD}(t, u)=2 \text {; if } r \text { is odd , }
$$

we have to calculate orbifold locus of weight 2 which may appear on $x$. In both cases we either get coordinate point of weight $r$ or $u$ which we already accounted for, so we do not get any new orbifold point. For the quasismoothness on the base locus, we instead use the 
computer algebra system to show that $X$ is quasismooth for $5 \leq r \leq 299$. Since base loci remains the same, we conclude that $X$ is quasismooth for all $n$.

3.2.8. Pfaffian Model 24. The paramter has the form $r=3 n, n \in \mathbb{N}$ and for a choice of input parameter $w=\frac{1}{2}(q, s, s, u, u)$ we get the ambient weighted projective variety

$$
{ }_{\mathrm{w}} \mathcal{G} \hookrightarrow \mathbb{P}\left(r^{2}, s^{3}, t^{4}, u\right) \text { with } K_{\mathrm{w}} \mathcal{G}=\mathcal{O}(-(5 r+7)) .
$$

We take a projective cone of weight 3 to get a 7 -fold $\mathcal{C}^{3} \mathrm{w} \mathcal{G}$ with the canonical divisor class $\mathcal{O}(-5(r+2))$. Then we take the intersection of this 7 -fold with two forms of weight $t$ and one form each of weight $u, s, r$ to get a Del Pezzo orbifold of index 2

$$
X:=\mathcal{C}^{3} \mathrm{w} \mathcal{G} \cap(t)^{2} \cap(u) \cap(s) \cap(r) \hookrightarrow \mathbb{P}\left(3, r, s^{2}, t^{2}\right)=\mathbb{P}(f, a, b, c, d, e) .
$$

The equations are given by the maximal Pfaffians of the skew symmetric matrix

$$
\left[\begin{array}{cccc}
a_{r} & H_{r} & b_{s} & H_{s} \\
& c_{s} & d_{t} & e_{t} \\
& & H_{t} & J_{t} \\
& & & H_{u}
\end{array}\right] .
$$

Quasismoothness: The details of orbifold loci lying on $X$ is summarized as follows.

\begin{tabular}{c|cc|l}
$P_{\text {orb }}$ & $X \cap P_{\text {orb }}$ & Tangent|local variables & Conditions on forms \\
& 2 points by 2.4 & $a, b, e \mid c, f$ & \\
$t$ & on patch $d$ & $a, c, d \mid b, f$ & $H_{r}=a+\cdots, H_{3}=b+\cdots$ \\
& on patch $e$ & $c, e, d \mid a, f$ & $H_{t}=d+\cdots, J_{t}=e+\cdots$ \\
$s$ & coordinate pt $b_{s}$ & $d, e, f \mid b, c$ & $H_{u}=a f+\cdots, H_{t}, J_{t}$ as for weight $t$ \\
$r$ & coordinate pt $a_{r}$ & $d, e, a \mid b, c$ & $H_{r}=f^{n}+\cdots, H_{u}=f^{n+1} a+\cdots$
\end{tabular}

The weight $t$ locus restricted to $X$ consists of 2 points. On each affine patch we get local variables of weight 3 and $s$ modulo $t$. The weight 3 locus is restricted to $X$ is given by

$$
V\left(a H_{u}, H_{r} H_{u}\right) \subset \mathbb{P}(3, r),
$$

giving 2 coordinate points of weight 3 and $r$. At the end, we may get singularities of weight 2 . If $r$ is even then the weight 2 locus is

$$
V\left(d J_{t}-e H_{t}, a H_{t}-d H_{r}, a J_{t}-e H_{r}\right) \subset \mathbb{P}\left[r, t^{2}\right],
$$

which gives 3 points. But there is no new singularity as we already got 2 points of type $\frac{1}{t}(3, s)$ and a point of type $\frac{1}{r}(1,1)$. If $r$ is odd, then it is a coordinate point of weight $s$ which we already accounted for and we do not get any new singularities. In this case the compute algebra computations run very fast and we verified the quasismoothness for $6 \leq r \leq 30,000$.

Remark 3.2. It is important to mention that the numerical candidate examples or models do not always give rise to quasismooth model of Del Pezzo surface. In the case of Fano index 2 , if we use the same numerical data as in the model $\mathrm{Pf}_{23}$ in 3.2 .7 for $r=3 n+1$. It appears to be an another model of families of orbifold Del Pezzo surfaces. It satisfies all 
the properties of the suggested model except quasismoothness on one affine patch of the weight 3 locus. We can not find 3 tangent monomials on the affine patch of weight $t$, so we do not include this model in our lists.

\section{4. $\mathbb{P}^{2} \times \mathbb{P}^{2}$ MODELS}

4.1. Generalities on $\mathrm{w}\left(\mathbb{P}^{2} \times \mathbb{P}^{2}\right)$. We first recall the definition of weighted $\mathbb{P}^{2} \times \mathbb{P}^{2}$ and formula for its Hilbert Series from BKQ18, Sze05] which we denote by w $\mathcal{P}$ for the rest of this article.

Definition 4.1. For a choice of two integer or half integer vectors $a=\left(a_{1}, a_{2}, a_{3}\right)$ and $b=\left(b_{1}, b_{2}, b_{3}\right)$ which satisfy

$$
a_{1}+b_{1}>0, a_{i} \leq a_{j} \text { and } b_{i} \leq b_{j} \text { for } 1 \leq i \leq j \leq 3,
$$

we define a $w \mathcal{P}$ as the quotient by $\mathbb{C}^{\times}$of the punctured affine cone $\widetilde{\mathcal{P} \backslash\{0\}}$ of the Segre embedding $\mathcal{P}=\mathbb{P}^{2} \times \mathbb{P}^{2} \hookrightarrow \mathbb{P}^{8}$ by

$$
\lambda: x_{i j} \mapsto \lambda^{a_{i}+b_{j}} x_{i j}, 1 \leq i, j \leq 3 .
$$

where the $x_{i j}$ are the coordinates of $\mathbb{P}^{8}$. Thus we get the embedding of

$$
\mathrm{w} \mathcal{P} \hookrightarrow \mathbb{P}^{8}\left(a_{1}+b_{1}, \ldots, a_{3}+b_{3}\right)
$$

for the choice of $a, b$, written together as a single input parameter $w=\left(a_{1}, a_{2}, a_{3} ; b_{1}, b_{2}, b_{3}\right)$. The equations are defined by $2 \times 2$ minors of the $3 \times 3$ matrix which we usually refer to as weighted matrix and write it as follows.

$$
\left(\begin{array}{lll}
x_{11} & x_{12} & x_{13} \\
x_{21} & x_{22} & x_{23} \\
x_{31} & x_{32} & x_{33}
\end{array}\right)=\left(\begin{array}{lll}
a_{1}+b_{1} & a_{1}+b_{2} & a_{1}+b_{3} \\
a_{2}+b_{1} & a_{2}+b_{2} & a_{2}+b_{3} \\
a_{3}+b_{1} & a_{3}+b_{2} & a_{3}+b_{3}
\end{array}\right)
$$

The Hilbert series of $w \mathcal{P}$ is given by

$$
P_{\mathrm{w} \mathcal{P}}(t)=\frac{1-\left(\sum_{1 \leq i, j \leq 3} t^{-\alpha_{i j}}\right) t^{d}+\left(4+\sum_{1 \leq i \neq j \leq 3} t^{\alpha_{j i}}+\sum_{1 \leq i \neq j \leq 3} t^{\beta_{j i}}\right) t^{d}-\left(\sum_{i, j} t^{\alpha_{i j}}\right) t^{d}+t^{2 d}}{\prod_{1 \leq i, j \leq 3}\left(1-t^{a_{i}+b_{j}}\right)},
$$

where $d=a_{1}+a_{2}+a_{3}+b_{1}+b_{2}+b_{3}, \alpha_{i j}=a_{i}+b_{j}, \alpha_{j i}=a_{i}-a_{j}$ and $\beta_{j i}=b_{i}-b_{j}$. If $\mathrm{w} \mathcal{P}$ is wellformed then the orbifold canonical class is given by

$$
K_{\mathrm{w} \mathcal{P}}=\mathcal{O}_{\mathrm{w} \mathcal{P}}\left(2 d-\sum_{i, j} a_{i}+b_{j}\right)=\mathcal{O}_{\mathrm{w} \mathcal{P}}(-d)
$$

Proposition 4.2. The degree of weighted $\mathbb{P}^{2} \times \mathbb{P}^{2}$ variety is given by

$$
\operatorname{deg}(\mathrm{w} \mathcal{P})=\frac{\left(\begin{array}{c}
2 d \\
4
\end{array}\right)+4\left(\begin{array}{l}
d \\
4
\end{array}\right)+\sum_{1 \leq i \neq j \leq 3}\left(\left(\begin{array}{c}
d+\beta_{j i} \\
4
\end{array}\right)+\left(\begin{array}{c}
d+\alpha_{j i} \\
4
\end{array}\right)\right)-\sum_{1 \leq i, j \leq 3}\left(\left(\begin{array}{c}
d+\alpha_{i j} \\
4
\end{array}\right)+\left(\begin{array}{c}
d-\alpha_{i j} \\
4
\end{array}\right)\right)}{\prod_{1 \leq i, j \leq 3}\left(a_{i}+b_{j}\right)}
$$


Then obviously if $X=\mathrm{w} \mathcal{P} \cap\left(\cap_{i=1}^{2}\left(g_{i}\right)\right)$ is complete intersection Del Pezzo surface of index $I$ then

$$
-K_{X}^{2}=I^{2} \prod_{i=1}^{2} \operatorname{deg}\left(g_{i}\right) \operatorname{deg}\left({ }_{\mathrm{w}} \mathcal{P}\right)
$$

4.2. Proof of Theorem 1.2. We prove the existence of each model with right invariants and singularities. Then we prove the quasi-smoothness of the each model. We write the proof for a general member $X$ of a family $\mathcal{X} \subset \mathbb{P}(a, b, c, d, e, f, g)$ of orbifold Del Pezzo surfaces. In the course of the proof our parameter is $r$ and the rest of the weights in terms of $r$ are

$$
\begin{array}{llll}
q=r-1, & s=r+1, & t=r+2, & u=r+3, \\
v=2 r+1, & y=2 r-2, & z=2 r-1, & m=3 r-2 .
\end{array}
$$

4.2.1. $\mathbb{P}^{2} \times \mathbb{P}^{2}$ model 11. The parameter $r=n, n \in \mathbb{N}$ and for an input parameter $w=(0,0, q ; 1,1, r)$, we get the embedding of a 4 dimensional orbifold

$$
\mathrm{w} \mathcal{P} \hookrightarrow \mathbb{P}\left(1^{4}, r^{4}, z\right) \text { with } K_{\mathrm{w} \mathcal{P}}=\mathcal{O}(-(2 r+1)) .
$$

Then if we take the complete intersection of 2 forms of degree $r$ then

$$
X:=\mathrm{w} \mathcal{P} \cap(r)^{2} \hookrightarrow \mathbb{P}\left(1^{4}, r^{2}, z\right)=\mathbb{P}(a, b, c, d, e, f, g)
$$

is a Del Pezzo surfaces of index 1.

The equations of $X$ can be described by the $2 \times 2$ minors of

$$
\left[\begin{array}{ccc}
a_{1} & b_{1} & c_{r} \\
d_{1} & e_{1} & H_{r} \\
f_{r} & J_{r} & g_{z}
\end{array}\right]
$$

Quasismoothness: Any member of the family of Del Pezzo orbifolds $X$ has two non-trivial orbifold strata, one is of weight $z$ and the other of weight $r$. The weight $z$ locus is just a coordinate point which obviously lies on $X$. The variables $a, b, d$ and $e$ serves as tangent variables to the variable $g$. Thus it is a point of type $\frac{1}{z}(1,1)$. On the other hand the weight $r$ locus is a copy restricted to $X$ is an empty set, so we do not get any other orbifolds singularities on $X$.

The base locus of linear system $|\mathcal{O}(r)|$ consists of just a coordinate point of weight $z$ which is manifestly quasismooth. Thus the each member in this model is a wellformed and quasismooth orbifold Del Pezzo surface with an orbifold point of type $\frac{1}{z}(1,1)$.

4.2.2. $\mathbb{P}^{2} \times \mathbb{P}^{2}$ model 12. The paramter has the form $r=2 n+1, n \in \mathbb{N}$ and for an input parameter $w=(0,1, q ; 1, r, s)$, we have the embedding

$$
\mathrm{w} \mathcal{P} \hookrightarrow \mathbb{P}\left(1,2, r^{2}, s^{2}, t, z, 2 r\right) \text { and } K_{\mathrm{w}} \mathcal{P}=\mathcal{O}(-(3 r+2)) .
$$

Then a $\log$ Del Pezzo surface of index 1 is obtained as the complete intersection of w $\mathcal{P}$ with a form degree $2 r$ and $s$ to get

$$
X:=\mathrm{w} \mathcal{P} \cap(2 r) \cap(s) \hookrightarrow \mathbb{P}\left(1,2, r^{2}, s, t, z\right)=\mathbb{P}(a, b, c, d, e, f, g) .
$$


Let $H_{s}$ and $H_{2 r}$ denote the the weighted homogeneous forms of degree $s$ and $2 r$ respectively, then the equations of $X$ are given by the $2 \times 2$ of

$$
\left[\begin{array}{ccc}
a_{1} & b_{2} & c_{r} \\
d_{r} & e_{s} & f_{z} \\
H_{s} & g_{t} & H_{2 r}
\end{array}\right] .
$$

Quasismoothness: The details of orbifold loci lying on $X$ is summarized as follows.

\begin{tabular}{cccl}
$P_{\text {orb }}$ & $X \cap P_{\text {orb }}$ & Tangent|local variables & Conditions on forms \\
\hline$z$ & coordinate pt $f_{z}$ & $a, b, g, e \mid c, d$ & $H_{s}=e+\cdots$ \\
$t$ & coordinate pt $g_{t}$ & $a, d, c, f \mid b, e$ & $F_{3}=d+\cdots$ \\
$r$ & coordinate pt $c_{r}$ & $d, e, g, a \mid b, f$ & $H_{s}=a c+\cdots$
\end{tabular}

The weight $s$ locus do not intersect $X$ as $H_{s}=e+\cdots$. The weight 2 locus consists of $\mathbb{P}[2, s]$ as $s$ is even but its an empty set as $H_{2 r}=b^{r}+\cdots$.

Moreover, $X$ may contain the orbifold locus of weight 5 as $X \cap \mathbb{P}[t, z]$, for example for $r=13$ but this does not give any new singularities at all. Similarly, $X$ may contain weight 3 locus as $X \cap \mathbb{P}[s, z]$; for example for $r=5$ but again this does not give any new singular point. Thus $X$ is a quasismooth on the orbifold locus. The quasismoothness on the base locus has been verified by computer algebra for $3 \leq n \leq 99$.

4.2.3. $\mathbb{P}^{2} \times \mathbb{P}^{2}$ model 13. The paramter has the form $r=2 n+1, n \in \mathbb{N}$ and for an input parameters $w=(0,1, q ; 1,2, r)$, we get the following embedding of the 4 -fold

$$
{ }_{\mathrm{w}} \mathcal{P} \hookrightarrow \mathbb{P}\left(1,2^{2}, 3, r^{2}, s^{2}, z\right) \text { and } K_{\mathrm{w} \mathcal{P}}=\mathcal{O}(-(2 r+3)) .
$$

The the complete intersection with two forms of degree $s$ is a log Del Pezzo surface

$$
X:={ }_{\mathrm{w}} \mathcal{P} \cap(s)^{2} \hookrightarrow \mathbb{P}\left(1,2^{2}, 3, r^{2}, z\right)=\mathbb{P}(a, b, c, d, e, f, g)
$$

of index 1 . The equations of $X$ are $2 \times 2$ minors of

$$
\left[\begin{array}{lll}
a_{1} & b_{2} & c_{r} \\
d_{2} & e_{3} & H_{s} \\
f_{r} & J_{s} & g_{z}
\end{array}\right] .
$$

Quasismoothness: The details of orbifold loci lying on $X$ is summarized as follows.

\begin{tabular}{c|cc|c}
$P_{\text {orb }}$ & $X \cap P_{\text {orb }}$ & Tangent|local variables & Conditions on forms \\
$z$ & coordinate pt $g_{z}$ & $a, b, d, e \mid c, f$ & \\
$r$ & 2 pts, on coordinate pt $c$ & $d, e, f, a \mid b, g$ & $J_{s}=a c+\cdots$ \\
3 & on coordinate pt $f$ & $b, c, e, a \mid d, g$ & $H_{s}=a f+\cdots$ \\
& coordinate pt $e_{3}$ & $a, c, f, g \mid b, d$ &
\end{tabular}

The weight 3 is a coordinate point if $r$ is not divisible by 3 . Otherwise, it defines 3 coordinate points of variables $c, e$ and $f$ and only the coordinate point of the variable $e$ 
gives a new orbifold point. if $z=0 \bmod 3$ then it gives only one new point. The weight 2 locus consists of $X \cap \mathbb{P}[b, d]$, which does not itesect $X$ as these variables also appear in $H_{s}$ and $J_{s}$. Thus $X$ is a quasismooth on the orbifold locus. The quasismoothness for this model has been verified by computer algebra for $3 \leq r \leq 37$.

4.2.4. $\mathbb{P}^{2} \times \mathbb{P}^{2}$ model 14. The paramter has the form $r=6 n-1, n \in \mathbb{N}$ and for input parameters $w=(0,1, q ; 2, r, s)$ to get

$$
\left.\mathrm{w} \mathcal{P} \hookrightarrow \mathbb{P}\left(2,3, r, s^{3}, t, z, 2 r\right) \text { with } K_{\mathrm{w}} \mathcal{G}=\mathcal{O}(-3(r+1))\right) .
$$

We take a projective cone of weight $r$ to get a 5 -fold $\mathcal{C}^{r}$ w $\mathcal{P}$ with the canonical divisor class $\mathcal{O}(-(4 r+3))$. Then the complete intersection of this 5 -fold with two forms of weight $s$ and one form of weight $2 r$ is a log Del Pezzo surface

$$
X:=\mathcal{C}^{3}{ }_{\mathrm{w}} \mathcal{P} \cap(s)^{2} \cap(2 r) \hookrightarrow \mathbb{P}(r, 2,3, r, s, t, z)=\mathbb{P}(g, a, b, c, d, e, f)
$$

of index 1 . The equations are given by $2 \times 2$ minors of

$$
\left[\begin{array}{ccc}
a_{2} & b_{3} & c_{s} \\
d_{r} & H_{s} & e_{z} \\
J_{s} & f_{t} & H_{2 r}
\end{array}\right],
$$

where $H_{s}, J_{s}$ and $H_{2 r}$ are forms of degree $s, s$ and $2 r$ respectively.

Quasismoothness: The details of orbifold loci lying on $X$ is summarized as follows.

\begin{tabular}{c|cc|l}
$P_{\text {orb }}$ & $X \cap P_{\text {orb }}$ & Tangent|local variables & Conditions on forms \\
$z$ & coordinate pt $e_{z}$ & $a, b, f, c \mid d, g$ & $G_{s}=c+\cdots$ \\
$t$ & coordinate pt $f_{t}$ & $a, c, d, e \mid b, g$ & \\
& 3 points by 2.4 & & \\
$r$ & on patch $d$ & $b, c, f, g \mid a, e$ & $H_{s}=c+\cdots, H_{2 r}=d g+\cdots$ \\
& on patch $g$ & $a, b, d, c \mid e, f$ & $H_{2 r}=g^{2}+\cdots, H_{s}=c+\cdots$ \\
3 & coordinate pt $b_{3}$ & $d, e, c, f \mid a, g$ & $H_{2 r}=b^{2 n} f+\cdots, F_{t}, G_{t}=c+\cdots$
\end{tabular}

The weight $s$ orbifold locus obviously does not intersect $X$. The weight 3 locus is $X \cap$ $\mathbb{P}[3, s, z]$ if $z=0 \bmod 3$ and $X \cap \mathbb{P}[3, s]$ otherwise. In both we only get the coordinate point of variable $b$ as a new orbifold point. Finally, the weight 2 locus is given by $X \cap \mathbb{P}[2, s]$ which obviously an empty set. Thus $X$ has the correct type of singularities and quasismooth on the orbifold locus. The quasismoothness has been verified by computer algebra for $5 \leq r \leq 611$.

4.3. Fano index 2 models. In this case we get two bi-parameterized models of families of $\log$ Del Pezzo surfaces with rigid singularities, i.e. indexed by $\mathbb{N} \times \mathbb{N}$. If we fix one parameter, we get a one parameter model which we computed earlier. Thus each of these two parameter models can be considered as consisting of infinite series of one parameter models.

Proof of Theorem 1.3; Let $r=3 m$ and $y=3 n+1$ be the two parameters with 
$q=r-1, s=r+1$ and $z=y+1$. If we choose the input parameter $(0,1, q ; 1,2, y)$ then we get the embedding of the ambient 4 dimensional orbifold w $\mathcal{P}$

$$
\mathrm{w} \mathcal{P} \hookrightarrow \mathbb{P}\left(1,2^{2}, 3, r, s, y, z, w\right),
$$

where $w=q+y$. The orbifold canonical class, computed from the Hilbert series, is given by

$$
K_{\mathrm{w} \mathcal{P}}=\mathcal{O}(-(r+y+3))
$$

Then the complete intersection with a form weight $w=r+y-1$ and a quadric gives a Del Pezzo surface of index 2:

$$
X=\mathrm{w} \mathcal{P} \cap\left(H_{w}\right) \cap\left(H_{2}\right) \hookrightarrow \mathbb{P}(1,2, r, 3, s, y, z)=\mathbb{P}(a, b, c, d, e, f, g) .
$$

The equations can be described by the $2 \times 2$ minors of the following matrix.

$$
\left[\begin{array}{ccc}
a_{1} & b_{2} & c_{r} \\
H_{2} & d_{3} & e_{s} \\
f_{y} & g_{z} & H_{w}
\end{array}\right] .
$$

Now we prove the quasi smoothness of each model one by one by the analysis of its orbifold loci.

4.3.1. $\mathbb{P}^{2} \times \mathbb{P}^{2}$ model 21. In this model the parameters $r$ and $y$ both are multiples of 3 . The details of orbifold loci lying on $X$ is summarized as follows.

\begin{tabular}{cccl}
$P_{\text {orb }}$ & $X \cap P_{\text {orb }}$ & Tangent $\mid$ local variables & Conditions on forms \\
\hline$z$ & coordinate pt $g_{z}$ & $a, c, e, b \mid d, f$ & $H_{2}=b+\cdots$ \\
$y$ & coordinate pt $f_{y}$ & $b, c, d, e \mid a, g$ & \\
$s$ & coordinate pt $e_{s}$ & $a, b, f, g \mid c, d$ & \\
$r$ & coordinate pt $c_{r}$ & $d, f, g, b \mid a, c$ & $H_{2}=b+\cdots$ \\
3 & coordinate pt $d_{3}$ & $a, c, f, b \mid e, g$ & $H_{w}=d^{m+n-1} b+\cdots$
\end{tabular}

The weight 3 is given by $X \cap \mathbb{P}[3, r, y]$ which gives 3 coordinate points. But only one new orbifold point appear on this locus as the coordinate points of weight $r$ and $y$ are counted earlier. The weight 2 locus is $X \cap \mathbb{P}[2, r, y]$ if $r$ and $y$ are even and is given by $X \cap \mathbb{P}[2, s, z]$ otherwise. Essentially we do not get any new orbifold since in each case we get $b \mathrm{H}_{2}$ as one of the defining equations of weight 2 locus. Thus $X$ is a quasismooth on the orbifold locus.

We verified the quasismoothness by using computer algebra for $6 \leq r, y \leq 63$.

4.3.2. $\mathbb{P}^{2} \times \mathbb{P}^{2}$ model 22. In this model we have $r=3 m, y=3 n+1$ such that $n \geq 2$ and $m \geq n$. The analysis of orbifold is similar for weight $z, y, s$ and $r$ to the firs model in the previous case. The weight 3 locus is given by $X \cap \mathbb{P}[3, r]$ which is given by

$$
V\left(d H_{w}, c d\right) \subset \mathbb{P}[c, d],
$$


since 3 divides $w$. This gives a coordinate point $c$ which is already considered as $\frac{1}{r}$ orbifold point. The weight 2 locus is $X \cap \mathbb{P}[2, r, z]$ if $r$ is even and $X \cap \mathbb{P}[2, s, y]$ otherwise. In both cases, we do not get any new orbifold point. Thus $X$ is a quasismooth on the orbifold locus. One can show that the base locus remains same for each value of parameter, following section 3.2.2. We used the computer algebra to verify the quasismoothness for $6 \leq r, y \leq 63$.

\section{Summary of COMPutational Results AND SPORADiC EXAMPles}

5.1. Summary of computational results. We use computer search routine of Qur17a, BKZ to search families of orbifold Del Pezzo surface with isolated orbifold points. The computer search is carried out in order of increasing the adjunction number of the Hilbert series of each format; separately for each Fano index. The computer search result do not have a termination condition but it returns complete list of candidate families for each adjunction number. Thus our results are complete up to a certain value of adjunction number. The Table 3 contains the summary of the computational results and details of sporadic families of log Del Pezzo surfaces. It contains the number of candidates from the computer search, how many of them contains only rigid singularities, how many of them are not in the models of the earlier sections $\$ 3$ and $\$ 4$ and how many of those sporadic cases are quasismooth.

TABle 3. The first column contains the format and the Fano index of these orbifold Del Pezzo surface. The column $q_{\max }$ gives the largest adjunction number searched in the given format; $\# X_{c}$ gives the number of candidates returned, $\# X_{r c}$ contains the number of candidates with only rigid singularities, $\# X_{s r c}$ contains the number sporadic rigid candidates (those candidates which are not in any models) and the last column \#QS$X_{\text {src }}$ gives the number of sporadic rigid families which are quasismooth.

\begin{tabular}{|c|c|c|c|c|c|}
\hline \hline Format- $I$ & $q_{\max }$ & $\# X_{c}$ & $\# X_{r c}$ & $\# X_{\text {src }}$ & $\#$ QS- $X_{\text {src }}$ \\
\hline $\mathrm{w} \mathcal{G}-1$ & 60 & 39 & 39 & 12 & 10 \\
\hline $\mathrm{w} \mathcal{G}-2$ & 52 & 116 & 46 & 29 & 6 \\
\hline $\mathrm{w} \mathcal{F}-1$ & 80 & 49 & 49 & 14 & 7 \\
\hline $\mathrm{w} \mathcal{F}-2$ & 68 & 127 & 44 & 5 & 0 \\
\hline
\end{tabular}

It is important to mention that there are other type of key varieties appearing in CR02, QS11, Qur15, Qur17b in codimension $c=5, \ldots, 10$ which can be used as ambient varieties to search for log Del Pezzo surfaces with isolated orbifold points. Indeed, we searched for the families of orbifold Del Pezzo surfaces in those formats but the computer search do not provide even sporadic examples. 
5.2. Sporadic cases. In this section we present those cases which are not in any of our models but appear as sporadic families of orbifold Del Pezzo surfaces in each format. We list the families of wellformed and quasismooth Del Pezzo surfaces with rigid singularities whos equations are those given by either the maximal Pfaffians of $5 \times 5$ skew symmetric matrix or by the $2 \times 2$ minors of order 3 matrices. Indeed, we follows all the steps of Section 2.3 to prove the existence, wellformedness and quasismoothness of the following families of orbifold Del Pezzo surfaces. In particular, we used computer algebra calculations to show the quasismoothness on base locus in these examples. We summarized the results in the form of the following table.

Table 4: $\log$ Del Pezzo surfaces in Pfaffian format

\begin{tabular}{|c|c|c|c|c|c|c|c|c|}
\hline WPS \& Para & Basket $\mathcal{B}$ & $-K_{X}^{2}$ & $h^{0}(-K)$ & $I$ & Wei & ght & & trix \\
\hline $\begin{array}{l}\mathbb{P}\left(1,3^{2}, 5^{2}, 7\right) \\
w=\frac{1}{2}(-1,3,3,7,11)\end{array}$ & $\frac{1}{3}(1,1), \frac{1}{5}(1,1), \frac{1}{7}(1,4)$ & $\frac{29}{105}$ & 1 & 1 & 1 & $\begin{array}{l}1 \\
3\end{array}$ & $\begin{array}{l}3 \\
5 \\
5\end{array}$ & $\begin{array}{l}5 \\
7 \\
7 \\
9\end{array}$ \\
\hline $\begin{array}{l}\mathbb{P}\left(3^{2}, 5^{2}, 7^{2}\right), \\
w=\frac{1}{2}(1,5,5,9,9)\end{array}$ & $3 \times \frac{1}{3}(1,1), \frac{1}{5}(1,1), 2 \times \frac{1}{7}(1,4)$ & $\frac{3}{35}$ & 0 & 1 & 3 & $\begin{array}{l}3 \\
5\end{array}$ & $\begin{array}{l}5 \\
7 \\
7\end{array}$ & $\begin{array}{l}5 \\
7 \\
7 \\
9\end{array}$ \\
\hline $\begin{array}{l}\mathbb{P}(1,3,5,7,9,11) \\
w=\frac{1}{2}(-1,3,7,11,15)\end{array}$ & $\frac{1}{3}(1,1), \frac{1}{11}(1,3)$ & $\frac{5}{33}$ & 1 & 1 & 1 & $\begin{array}{l}3 \\
5\end{array}$ & $\begin{array}{l}5 \\
7 \\
9\end{array}$ & $\begin{array}{c}7 \\
9 \\
11 \\
13\end{array}$ \\
\hline $\begin{array}{l}\mathbb{P}(3,5,6,7,8,13) \\
w=\frac{1}{2}(1,5,9,11,15)\end{array}$ & $2 \times \frac{1}{3}(1,1), \frac{1}{7}(1,4), \frac{1}{13}(1,9)$ & $\frac{11}{273}$ & 0 & 1 & 3 & $\begin{array}{l}5 \\
7\end{array}$ & $\begin{array}{c}6 \\
8 \\
10\end{array}$ & $\begin{array}{c}8 \\
10 \\
12 \\
13\end{array}$ \\
\hline $\begin{array}{l}\mathbb{P}(1,4,5,7,11,17) \\
w=(0,1,4,7,10)\end{array}$ & $\frac{1}{17}(1,4)$ & $\frac{2}{17}$ & 1 & 1 & 1 & $\begin{array}{l}4 \\
5\end{array}$ & $\begin{array}{c}7 \\
8 \\
11\end{array}$ & $\begin{array}{l}10 \\
11 \\
14 \\
17\end{array}$ \\
\hline $\begin{array}{l}\mathbb{P}(3,5,7,9,11,13), \\
w=\frac{1}{2}(3,7,11,11,15)\end{array}$ & $\frac{1}{3}(1,1), \frac{1}{5}(1,2) \frac{1}{7}(1,4), \frac{1}{13}(1,8)$ & $\frac{41}{1365}$ & 0 & 1 & 5 & $\begin{array}{l}7 \\
7\end{array}$ & $\begin{array}{c}7 \\
9 \\
11\end{array}$ & $\begin{array}{c}9 \\
11 \\
13 \\
15\end{array}$ \\
\hline $\begin{array}{l}\mathbb{P}(4,5,7,10,11,13) \\
w=\frac{1}{2}(1,7,9,13,19)\end{array}$ & $\frac{1}{5}(1,2), \frac{1}{11}(1,8), \frac{1}{13}(1,9)$ & $\frac{16}{715}$ & 0 & 1 & 4 & $\begin{array}{l}5 \\
8\end{array}$ & $\begin{array}{c}7 \\
10 \\
11\end{array}$ & $\begin{array}{l}10 \\
13 \\
14 \\
16\end{array}$ \\
\hline
\end{tabular}




\begin{tabular}{|c|c|c|c|c|c|c|c|c|}
\hline $\begin{array}{l}\mathbb{P}(1,3,7,8,13,19), \\
w=(-2,3,5,9,10)\end{array}$ & $\frac{1}{7}(1,4), \frac{1}{13}(1,9), \frac{1}{19}(1,10)$ & $\frac{191}{1729}$ & 0 & 1 & 1 & $\begin{array}{l}3 \\
8\end{array}$ & $\begin{array}{c}7 \\
12 \\
14\end{array}$ & $\begin{array}{c}8 \\
13 \\
15 \\
19\end{array}$ \\
\hline $\begin{array}{l}\mathbb{P}(1,5,6,9,14,19) \\
w=\frac{1}{2}(-3,5,13,15,23)\end{array}$ & $\frac{1}{9}(1,1), \frac{1}{19}(1,15)$ & $\frac{13}{171}$ & 1 & 1 & 1 & $\begin{array}{l}5 \\
9\end{array}$ & $\begin{array}{c}6 \\
10 \\
14\end{array}$ & $\begin{array}{l}10 \\
14 \\
18 \\
19\end{array}$ \\
\hline $\begin{array}{l}\mathbb{P}(1,5,7,10,14,23) \\
w=\frac{1}{2}(-1,3,11,17,19)\end{array}$ & $\frac{1}{5}(1,2), \frac{1}{7}(1,4), \frac{1}{23}(1,6)$ & $\frac{52}{805}$ & 1 & 1 & 1 & $\begin{array}{l}5 \\
7\end{array}$ & $\begin{array}{c}8 \\
10 \\
14\end{array}$ & $\begin{array}{l}14 \\
16 \\
20 \\
23\end{array}$ \\
\hline $\begin{array}{l}\mathbb{P}(1,3,5,6,7,8), \\
w=(0,1,2,5,6)\end{array}$ & $\frac{1}{3}(1,1), \frac{1}{5}(1,1), \frac{1}{8}(1,5)$ & $\frac{19}{30}$ & 1 & 2 & 1 & $\begin{array}{l}2 \\
3\end{array}$ & $\begin{array}{l}5 \\
6 \\
7\end{array}$ & $\begin{array}{c}6 \\
7 \\
8 \\
11\end{array}$ \\
\hline $\begin{array}{l}\mathbb{P}\left(4,5^{2}, 7^{2}, 8\right), \\
w=(2,2,3,5,5)\end{array}$ & $2 \times \frac{1}{5}(1,3), 2 \times \frac{1}{7}(1,3), \frac{1}{8}(1,1)$ & $\frac{11}{70}$ & 0 & 2 & 4 & $\begin{array}{l}5 \\
5\end{array}$ & $\begin{array}{l}7 \\
7 \\
8\end{array}$ & $\begin{array}{c}7 \\
7 \\
8 \\
10\end{array}$ \\
\hline $\begin{array}{l}\mathbb{P}\left(3,6,7^{2}, 8^{2}\right), \\
w=\frac{1}{2}(5,7,7,9,9)\end{array}$ & $\frac{1}{3}(1,1), \frac{1}{6}(1,1), \frac{1}{7}(1,2), 2 \times \frac{1}{8}(1,5)$ & $\frac{1}{7}$ & 0 & 2 & 6 & $\begin{array}{l}6 \\
7\end{array}$ & $\begin{array}{l}7 \\
8 \\
8\end{array}$ & $\begin{array}{l}7 \\
8 \\
8 \\
9\end{array}$ \\
\hline $\begin{array}{l}\mathbb{P}\left(3,6,7^{2}, 8,11\right), \\
w=(1,2,5,6,6)\end{array}$ & $2 \times \frac{1}{3}(1,1), \frac{1}{7}(1,2), \frac{1}{8}(1,5), \frac{1}{11}(1,3)$ & $\frac{59}{462}$ & 0 & 2 & 3 & $\begin{array}{l}6 \\
7\end{array}$ & $\begin{array}{c}7 \\
8 \\
11\end{array}$ & $\begin{array}{c}7 \\
8 \\
11 \\
12\end{array}$ \\
\hline $\begin{array}{l}\mathbb{P}\left(3,7,8^{2}, 9,10\right), \\
w=\frac{1}{2}(7,7,9,9,11)\end{array}$ & $\frac{1}{3}(1,1), \frac{1}{7}(1,1), 2 \times \frac{1}{8}(1,5), \frac{1}{10}(1,3)$ & $\frac{11}{105}$ & 0 & 2 & 7 & $\begin{array}{l}8 \\
8\end{array}$ & $\begin{array}{l}8 \\
8 \\
9\end{array}$ & $\begin{array}{c}9 \\
9 \\
10 \\
10\end{array}$ \\
\hline $\begin{array}{l}\mathbb{P}\left(4,7^{2}, 9^{2}, 12\right) \\
w=(2,2,5,7,7)\end{array}$ & $2 \times \frac{1}{7}(1,3), 2 \times \frac{1}{9}(1,4), \frac{1}{12}(1,1)$ & $\frac{5}{63}$ & 0 & 2 & 4 & $\begin{array}{l}7 \\
7\end{array}$ & $\begin{array}{c}9 \\
9 \\
12\end{array}$ & $\begin{array}{c}9 \\
9 \\
12 \\
14\end{array}$ \\
\hline
\end{tabular}


Table 5: $\log$ Del Pezzo surfaces in $\mathbb{P}^{2} \times \mathbb{P}^{2}$ format

\begin{tabular}{|c|c|c|c|c|c|c|c|}
\hline WPS, Para & Basket $\mathcal{B}$ & $-K_{X}^{2}$ & $h^{0}(-K)$ & $I$ & Weigh & it $\mathrm{N}$ & Matrix \\
\hline $\begin{array}{l}\mathbb{P}\left(1,2,3^{2}, 4,5^{2}\right) \\
w=(0,1,2 ; 1,3,4)\end{array}$ & $\frac{1}{3}(1,1), \frac{1}{5}(1,2), \frac{1}{5}(1,1)$ & $\frac{8}{15}$ & 1 & 1 & $\begin{array}{l}1 \\
3 \\
4\end{array}$ & $\begin{array}{l}2 \\
4 \\
5\end{array}$ & $\begin{array}{l}3 \\
5 \\
6\end{array}$ \\
\hline $\begin{array}{l}\mathbb{P}\left(3,4,5^{2}, 6,7^{2}\right) \\
w=(0,1,2 ; 4,5,6)\end{array}$ & $2 \times \frac{1}{5}(1,2), 2 \times \frac{1}{7}(1,4)$ & $\frac{3}{35}$ & 0 & 1 & $\begin{array}{l}4 \\
5 \\
6\end{array}$ & $\begin{array}{l}5 \\
6 \\
7\end{array}$ & $\begin{array}{l}6 \\
7 \\
8\end{array}$ \\
\hline $\begin{array}{l}\mathbb{P}\left(2,3,5^{2}, 6,9,13\right) \\
w=(0,1,4 ; 2,5,9)\end{array}$ & $2 \times \frac{1}{3}(1,1), 2 \times \frac{1}{5}(1,2), \frac{1}{5}(1,1), \frac{1}{13}(1,7)$ & $\frac{22}{195}$ & 0 & 1 & $\begin{array}{l}2 \\
5 \\
9\end{array}$ & $\begin{array}{c}3 \\
6 \\
10\end{array}$ & $\begin{array}{c}6 \\
9 \\
13\end{array}$ \\
\hline $\begin{array}{l}\mathbb{P}\left(1,3,5^{2}, 7,9,13\right) \\
w=(0,2,4 ; 1,5,9)\end{array}$ & $\frac{1}{5}(1,2), \frac{1}{5}(1,1), \frac{1}{7}(1,4), \frac{1}{13}(1,7)$ & $\frac{86}{455}$ & 1 & 1 & $\begin{array}{l}1 \\
3 \\
5\end{array}$ & $\begin{array}{l}5 \\
7 \\
9\end{array}$ & $\begin{array}{c}9 \\
11 \\
13\end{array}$ \\
\hline $\begin{array}{l}\mathbb{P}\left(1,5^{2}, 7,9,11,17\right) \\
w=(0,4,6 ; 1,5,11)\end{array}$ & $\frac{1}{5}(1,2), \frac{1}{7}(1,4), \frac{1}{9}(1,1), \frac{1}{17}(1,4)$ & $\frac{562}{5355}$ & 1 & 1 & $\begin{array}{c}1 \\
5 \\
11\end{array}$ & $\begin{array}{c}5 \\
9 \\
15\end{array}$ & $\begin{array}{c}7 \\
11 \\
17\end{array}$ \\
\hline $\begin{array}{l}\mathbb{P}\left(2,3,7^{2}, 8,13,19\right) \\
w=(0,1,6 ; 2,7,13)\end{array}$ & $\frac{1}{3}(1,1), 2 \times \frac{1}{7}(1,3), \frac{1}{7}(1,4), \frac{1}{19}(1,12)$ & $\frac{4}{57}$ & 0 & 1 & $\begin{array}{c}2 \\
7 \\
13\end{array}$ & $\begin{array}{c}3 \\
8 \\
14\end{array}$ & $\begin{array}{c}8 \\
13 \\
19\end{array}$ \\
\hline
\end{tabular}

\section{REFERENCES}

$\left[\mathrm{ACC}^{+} 16\right]$ Mohammad Akhtar, Tom Coates, Alessio Corti, Liana Heuberger, Alexander Kasprzyk, Alessandro Oneto, Andrea Petracci, Thomas Prince, and Ketil Tveiten, Mirror symmetry and the classification of orbifold Del Pezzo surfaces, Proceedings of the American Mathematical Society 144 (2016), no. 2, 513-527.

[AK14] Mohammad Akhtar and Alexander Kasprzyk, Singularity content, arXiv preprint arXiv:1401.5458 (2014).

[BKQ18] Gavin Brown, Alexander M. Kasprzyk, and Muhammad Imran Qureshi, Fano 3-folds in $\mathbb{P}^{2} \times \mathbb{P}^{2}$ format, Tom and Jerry, European Journal of Mathematics 4 (2018), no. 1, 51-72.

[BKZ] G. Brown, A. M. Kasprzyk, and L. Zhu, Gorenstein formats, canonical and Calabi-Yau threefolds, arXiv:1409.4644.

[BRZ13] A. Buckley, M. Reid, and S. Zhou, Ice cream and orbifold Riemann-Roch, Izvestiya: Mathematics 77:3 (2013), 461-486.

[BZ10] Gavin Brown and Francesco Zucconi, Graded rings of rank 2 Sarkisov links, Nagoya Mathematical Journal 197 (2010), 1-44.

$\left[\mathrm{CCG}^{+} 12\right]$ Tom Coates, Alessio Corti, Sergey Galkin, Vasily Golyshev, and Alexander Kasprzyk, Mirror symmetry and Fano manifolds, Proceedings of European Congress of Mathematics (Krakow, 2-7 July, 2012), January 2014 (824 pages), ISBN 978-3-03719-120, DOI 10.4171/120-1/16 (2012).

[CH17] Alessio Corti and Liana Heuberger, Del Pezzo surfaces with $\frac{1}{3}(1,1)$ points, Manuscripta Math. 153 (2017), no. 1-2, 71-118.

[CP17] Daniel Cavey and Thomas Prince, Del Pezzo surfaces with a single $1 / k(1,1)$ singularity, arXiv preprint arXiv:1707.09213 (2017). 
[CPS10] Ivan Cheltsov, Jihun Park, and Constantin Shramov, Exceptional Del Pezzo hypersurfaces, Journal of Geometric Analysis 20 (2010), no. 4, 787-816.

[CR02] A. Corti and M. Reid, Weighted Grassmannians, Algebraic geometry (M. C. Beltrametti, F. Catanese, C. Ciliberto, A. Lanteri, and C. Pedrini, eds.), de Gruyter, Berlin, 2002, pp. 141163.

[CS13] Ivan Cheltsov and Constantin Shramov, Del Pezzo zoo, Experimental Mathematics 22 (2013), no. 3, 313-326.

[IF00] A. R. Iano-Fletcher, Working with weighted complete intersections, Explicit Birational Geometry of 3-folds, vol. 281, London Math. Soc. Lecture Note Ser, CUP, 2000, pp. 101-173.

[JK01] Jennifer M Johnson and János Kollár, Kähler-Einstein metrics on log Del Pezzo surfaces in weighted projective 3-spaces, Annales de l'institut Fourier, vol. 51, 2001, pp. 69-79.

[KMM87] Yujiro Kawamata, Katsumi Matsuda, and Kenji Matsuki, Introduction to the minimal model problem, Adv. Stud. Pure Math 10 (1987), 283-360.

[KP15] In-Kyun Kim and Jihun Park, Log canonical thresholds of complete intersection log Del Pezzo surfaces, Proceedings of the Edinburgh Mathematical Society 58 (2015), no. 2, 445-483.

[KSB88] János Kollár and Nicholas I Shepherd-Barron, Threefolds and deformations of surface singularities, Inventiones mathematicae 91 (1988), no. 2, 299-338.

[May16] Evgeny Mayanskiy, Weighted complete intersection Del Pezzo surfaces, arXiv preprint arXiv:1608.02049 (2016).

[QS11] M. I. Qureshi and B. Szendrői, Constructing projective varieties in weighted flag varieties, Bull. Lon. Math Soc. 43 (2011), no. 2, 786-798.

[QS12] M. I. Qureshi and Balázs Szendrői, Calabi-Yau threefolds in weighted flag varieties, Adv. High Energy Phys. (2012), Art. ID 547317, 14 pp.

[Qur15] M. I. Qureshi, Constructing projective varieties in weighted flag varieties II, Math. Proc. Camb. Phil. Soc. 158 (2015), 193-209.

[Qur17a] Muhammad Imran Qureshi, Computing isolated orbifolds in weighted flag varieties, Journal of Symbolic Computation 79, Part 2 (2017), 457 - 474.

[Qur17b] _ Polarized 3-folds in a codimension 10 weighted homogeneous f4 variety, Journal of Geometry and Physics 120 (2017), 52 - 61.

[Sze05] Balázs Szendrői, On weighted homogeneous varieties, Unpublished manuscript, 2005.

Muhammad Imran Qureshi, Department of Mathematics, SBASSE Lahore University of Management Sciences (LUMS) Lahore, Pakistan and Mathematisches Institut, Universität TüBingen, Germany

E-mail address: i.qureshi@maths.oxon.org 